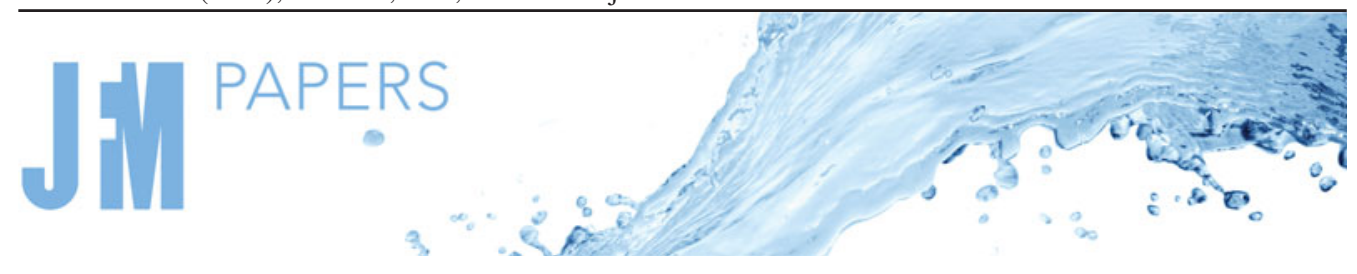

\title{
Effects of clouds and phase changes on fast-wave averaging: a numerical assessment
}

\author{
Yeyu Zhang ${ }^{1, \dagger}$, Leslie M. Smith ${ }^{1,2}$ and Samuel N. Stechmann ${ }^{1,3}$ \\ ${ }^{1}$ Department of Mathematics, University of Wisconsin-Madison, WI 53706, USA \\ ${ }^{2}$ Department of Engineering Physics, University of Wisconsin-Madison, WI 53706, USA \\ ${ }^{3}$ Department of Atmospheric and Oceanic Sciences, University of Wisconsin-Madison, WI 53706, USA
}

(Received 22 August 2020; revised 21 March 2021; accepted 13 May 2021)

A remarkable property of geophysical fluids is that, even for nonlinear flows, a slow component can sometimes evolve independently of the fast-wave components. The dry Boussinesq equations, for instance, are known to exhibit this property for small Froude $(\mathrm{Fr})$ and Rossby $(\mathrm{Ro})$ numbers (i.e. strong stratification and rapid rotation). Here, we ask: Do the moist Boussinesq equations also exhibit this property, even if clouds are included as changes of water between different phases (vapour and liquid)? To investigate, the authors recently performed an asymptotic analysis and identified several ways in which phase changes could possibly induce coupling between the slow component and fast waves; however, these possibilities were not clearly settled from theoretical considerations alone. Here, to investigate further, a suite of numerical simulations is conducted, using a sequence of small values $F r=\operatorname{Ro}=1,10^{-1}, 10^{-2}, 10^{-3}$. For $\operatorname{Fr}=\operatorname{Ro}=10^{-1}$, the influence of waves on the slow component is relatively small, but does not decrease proportional to $\mathrm{Fr}$ and $\mathrm{Ro}$, as $\mathrm{Fr}$ and $\mathrm{Ro}$ are decreased to $10^{-2}$ and $10^{-3}$. As an explanation and physical interpretation, it is shown that, while linear waves have a time average of zero, the piecewise-linear waves that arise due to phase changes actually have a non-zero time-averaged component.

Key words: condensation/evaporation, computational methods, atmospheric flows

\section{Introduction}

As a model of atmospheric or oceanic dynamics, the dry Boussinesq equations have a remarkable property: for small values of the Froude and Rossby numbers, the state vector

$\dagger$ Present address: School of Mathematics, Shanghai University of Finance and Economics, Shanghai 200433, PR China. Email address for correspondence: yzhang676@wisc.edu

(C) The Author(s), 2021. Published by Cambridge University Press. This is an Open Access article, distributed under the terms of the Creative Commons Attribution-NonCommercial-ShareAlike licence (http://creativecommons.org/licenses/by-nc-sa/4.0/), which permits non-commercial re-use, distribution, and reproduction in any medium, provided the same Creative Commons licence is included and the original work is properly cited. The written permission of Cambridge University 


\section{Y. Zhang, L.M. Smith and S.N. Stechmann}

can be decomposed into a slow vortical component and a fast-wave component, and the slow component obeys its own evolution equation, independent of the dynamics of the fast inertio-gravity waves (Embid \& Majda 1996, 1998; Majda \& Embid 1998; Majda 2003). In a sense, then, in considering the evolution of the slow component, the effects of the fast waves are averaged out. In earlier work, a similar type of fast-wave averaging property was also shown for compressible fluid dynamics, for a small Mach number, where the fast waves correspond to acoustic/sound waves (Klainerman \& Majda 1981, 1982; Majda 1984). Many other examples also arise from fluid dynamics, such as the rotating shallow water equations, and all of these examples fall under the category of fast singular limits of hyperbolic partial differential equations (PDEs), with unbalanced initial conditions, which have been the topic of numerous other studies as well (e.g. Schochet 1994; Babin, Mahalov \& Nicolaenko 1996; Babin et al. 1997; Babin, Mahalov \& Nicolaenko 1998, 2000; Schochet 2005; Dutrifoy \& Majda 2006, 2007; Dutrifoy, Majda \& Schochet 2009; Wingate et al. 2011).

Here, the main question is: What happens if moisture, clouds and phase changes are included? Does the slow component still evolve essentially independently of the fast-wave component? Or, do clouds and phase changes create new types of coupling between the slow and fast components?

To investigate these questions, we use a fast-wave averaging framework that was recently presented to include the additional effects of clouds and phase changes (Zhang, Smith \& Stechmann 2021). In this case, several new challenges arise, and one of the most significant challenges is that, while the dry case includes a constant coefficient linear operator, the phase-change case includes an operator that is spatially and temporally varying. Due to this additional complexity, while Fourier methods are available for use in the dry case, they are no longer amenable in the case with clouds and phase changes. As a result, the formal asymptotic analysis of Zhang et al. (2021) includes some terms that are difficult to probe analytically. In the present paper, to investigate further, the fast-wave averaging framework is studied using numerical simulations.

The present study can also be viewed as an investigation of wave and vortical interactions, a topic that has a long history in the context of the dry Boussinesq equations and related systems of geophysical fluid dynamics (e.g. Phillips 1968; Greenspan 1969; Lelong \& Riley 1991; Bartello 1995; Embid \& Majda 1996; Babin et al. 1996, 1997; Embid \& Majda 1998; Majda \& Embid 1998; Smith \& Waleffe 1999; Babin et al. 1998, 2000; Smith \& Waleffe 2002; Majda 2003; Remmel \& Smith 2009; Wingate et al. 2011). In some studies of wave-vortical interactions, the main topic of interest is the statistical properties of forced turbulence or turbulent decay. Note that the present paper focuses instead on initial value problems, as such a set-up is most directly in line with the fast-wave averaging framework. It would be interesting to investigate statistical properties of turbulence in the future.

In the limit of small Rossby and Froude numbers (large rotation and stratification, respectively), it is the quasi-geostrophic (QG) equations that describe the evolution of the slow, vortical mode. Two cases should be distinguished, according to the initial conditions (e.g. Klainerman \& Majda 1981, 1982; Majda 1984, 2003). On the one hand, if the initial conditions contain no waves (or if the waves are sufficiently small in amplitude or norm), it is said that the initial data are balanced or well-prepared. In this case, the solutions of the Boussinesq equations will converge to solutions of the QG equations. On the other hand, if the initial conditions are general and contain wave contributions, it is said that the initial data are unbalanced or ill-prepared. This latter case is where fast-wave averaging is relevant. Remarkably, even for unbalanced initial conditions, the QG equations describe 


\section{Effects of clouds and phase changes on fast-wave averaging}

the limiting dynamics of the slow modes, and the fast waves are also present in the limit but do not influence the QG evolution.

While much is known about dry dynamics without moisture (for either balanced or unbalanced initial conditions), much less is known about moist dynamics with phase changes. In the case of balanced initial conditions, with moisture, a formal asymptotic derivation of precipitating QG (PQG) equations has been presented (Smith \& Stechmann 2017), and some properties of the PQG equations have been investigated (Wetzel, Smith \& Stechmann 2017, 2019a; Edwards, Smith \& Stechmann 2020a,b), but no rigorous proofs have been shown. Unbalanced initial conditions, on the other hand, are the topic of the present paper. Some main questions are: Do the PQG equations describe the evolution of the slow modes, in the limit of small Froude and Rossby numbers, even if the initial conditions are unbalanced? Is the slow-mode evolution influenced by waves, or not?

The investigation here also contributes to the growing body of literature on moist dynamics of the atmosphere, including analytical studies such as mathematically rigorous results (e.g. Majda \& Souganidis 2010; Zelati \& Temam 2012; Zelati et al. 2013; Bousquet, Zelati \& Temam 2014; Zelati et al. 2015; Li \& Titi 2016; Hittmeir et al. 2017; Cao et al. 2018; Hittmeir et al. 2020) and asymptotic analysis (e.g. Klein \& Majda 2006; Majda 2007; Khouider, Majda \& Stechmann 2013; Chen, Majda \& Stechmann 2015, 2016; Smith \& Stechmann 2017; Hittmeir \& Klein 2018; Rosemeier, Baumgartner \& Spichtinger 2018), as well as computational studies of moist turbulence (e.g. Spyksma, Bartello \& Yau 2006; Schumacher \& Pauluis 2010; Sukhatme, Majda \& Smith 2012). The atmosphere in nature includes the effects of moisture and changes of water between different phases (vapour, liquid, etc.), and this growing literature is helping to shrink the gap between our understanding of dry versus moist atmospheric dynamics.

The remainder of the paper is organized as follows. Background information is presented in $\S \S 2$ and 3, including the equations of motion (the moist Boussinesq equations with phase changes) and a summary of the asymptotic theory of fast-wave averaging $(\$ 2)$, followed by a description of the set-up of numerical simulations and data analysis methods (§3). The numerical investigation of fast-wave averaging is then presented in $\S 4$ and is aimed at the main questions of the paper, such as: Do the slow modes still evolve independently of the fast waves, even in the presence of phase changes? Following the numerical experiments, an explanation and physical interpretation are described in $\S 5$. Conclusions are discussed in $\S 6$.

\section{Theoretical background}

The model equations are described here in $\S 2.1$. First, we present the equations in a way that highlights the new features of moisture and phase changes, beyond the simpler case of dry dynamics. Then the equations are rewritten in a different way, using conserved thermodynamic variables, in order to facilitate the definition of the slow variables in $\S 2.2$. Subsequent subsections describe the fast-wave averaging framework.

\subsection{The dynamical equations}

In past studies of fast-wave averaging (e.g. Embid \& Majda 1996), the dry Boussinesq equations have been investigated. Here, instead, moist Boussinesq equations are investigated, in order to assess the impact of phase changes between water vapour and liquid water, following Zhang et al. (2021). The governing equations are

$$
\frac{\mathrm{D} u}{\mathrm{D} t}+f \hat{z} \times \boldsymbol{u}=-\nabla \phi+\hat{z} b,
$$




$$
\begin{gathered}
\nabla \cdot \boldsymbol{u}=0, \\
\frac{\mathrm{D} \theta}{\mathrm{D} t}+w \frac{\mathrm{d} \tilde{\theta}}{\mathrm{d} z}=\frac{L_{v}}{c_{p}} C, \\
\frac{\mathrm{D} q_{v}}{\mathrm{D} t}+w \frac{\mathrm{d} \tilde{q}_{v}}{\mathrm{~d} z}=-C, \\
\frac{\mathrm{D} q_{l}}{\mathrm{D} t}=C .
\end{gathered}
$$

Here, $\boldsymbol{u}=\left(\boldsymbol{u}_{h}, w\right)$ is the three-dimensional velocity with horizontal components $\boldsymbol{u}_{h}=$ $(u, v)$ and vertical component $w$. The material derivative is defined as $\mathrm{D} / \mathrm{D} t=\partial_{t}+\boldsymbol{u} \cdot \boldsymbol{\nabla}$, where the three-dimensional gradient operator is $\nabla=\left(\partial_{x}, \partial_{y}, \partial_{z}\right)$. The conservation of momentum equation is in (2.1), including the Coriolis term, $f \hat{z} \times \boldsymbol{u}=(-f v, f u, 0)^{\top}$, which represents the effects of rotation. The unit vector in the vertical direction is $\hat{z}=(0,0,1)$ and the pressure-like variable is $\phi$. A constant Coriolis parameter $f$ is used here.

The buoyancy term $\hat{z} b$ in (2.1) is defined as

$$
b=g\left(\frac{\theta}{\theta_{0}}+R_{v d} q_{v}-q_{l}\right),
$$

where $g=9.8 \mathrm{~m} \mathrm{~s}^{-2}$ is the gravitational acceleration, $\theta_{0}=300 \mathrm{~K}$ is a reference background value of potential temperature, and $R_{v d}=\left(R_{v} / R_{d}\right)-1 \approx 0.61$, where $R_{d}$ is the gas constant for dry air and $R_{v}$ is the gas constant for water vapour.

Three thermodynamic variables are evolving in time according to (2.3)-(2.5). The potential temperature is $\theta$, the water vapour mixing ratio is $q_{v}$ and the liquid water mixing ratio is $q_{l}$, and all three of these quantities are anomalies. More specifically, the thermodynamic quantities have been decomposed as $\theta^{t o t}(\boldsymbol{x}, t)=\tilde{\theta}(z)+\theta(\boldsymbol{x}, t)$ and $q_{v}^{t o t}(\boldsymbol{x}, t)=\tilde{q}_{v}(z)+q_{v}(\boldsymbol{x}, t)$, so that the total quantity is written as the sum of a prescribed, background function of altitude $z$ and an anomaly. Note that the background state is chosen to be cloud-free, so $\tilde{q}_{l}=0$ and $q_{l}^{\text {tot }}=q_{l}$. Furthermore, the background gradients $d \tilde{\theta} / \mathrm{d} z$ and $\mathrm{d} \tilde{q}_{v} / \mathrm{d} z$ are chosen to be constants, for simplicity, which helps to render (2.1)-(2.5) a constant-coefficient system within each phase, and a piecewise-constant-coefficient system overall, since some coefficients change their values due to phase changes.

Phase changes enter into (2.3)-(2.5) via $C$, which represents the rate of condensation and evaporation. Condensation occurs for $C>0$ and represents a phase transition from vapour to liquid, and evaporation occurs for $C<0$ and represents a phase transition from liquid to vapour. The definition of $C$ is

$$
C= \begin{cases}0, & \text { if } q_{v}^{t o t}<q_{v s}^{t o t}, \\ -\frac{\mathrm{D} q_{v s}^{t o t}}{\mathrm{D} t}, & \text { if } q_{v}^{t o t}=q_{v s}^{t o t},\end{cases}
$$

where the threshold $q_{v s}^{\text {tot }}$ is the saturation water vapour mixing ratio. In other words, in (2.7), if the vapour is below the threshold, then neither condensation nor evaporation occurs; and if the vapour reaches the threshold, then condensation or evaporation will occur (so $C \neq 0$ ) and is defined so as to maintain the vapour value at its threshold value: $q_{v}^{t o t}=q_{v s}^{t o t}$. Indeed, from inserting (2.7) into (2.4), one can see that, if $q_{v}^{t o t}=q_{v s}^{t o t}$, then $D q_{v}^{\text {tot }} / D t=D q_{v s}^{\text {tot }} / D t$. To close the evolution, the threshold $q_{v s}^{\text {tot }}$ must be specified. In comprehensive treatments of moist thermodynamics, one would define $q_{v s}^{\text {tot }}$ to be a 


\section{Effects of clouds and phase changes on fast-wave averaging}

function of temperature and pressure, according to the Clausius-Clapeyron equation (e.g. Rogers \& Yau 1989; Grabowski \& Smolarkiewicz 1996; Klein \& Majda 2006). Here, as a more simplistic treatment, it is defined as a function of height $z$ alone, as $q_{v s}^{\text {tot }}(z)$. Such a treatment arises from assuming that temperature $T^{\text {tot }}$ and pressure $p^{\text {tot }}$ are nearly equal to their background values $\tilde{T}(z)$ and $\tilde{p}(z)$, in which case $q_{v s}^{t o t}\left(T^{t o t}, p^{t o t}\right) \approx q_{v s}^{t o t}(\tilde{T}(z), \tilde{p}(z))$ as a first approximation (e.g. Hernandez-Duenas et al. 2013). In this case, with $q_{v s}^{\text {tot }}=q_{v s}^{\text {tot }}(z)$, (2.7) becomes

$$
C= \begin{cases}0, & \text { if } q_{v}^{t o t}<q_{v s}^{t o t}, \\ -w \frac{\mathrm{d} q_{v s}^{t o t}(z)}{\mathrm{d} z}, & \text { if } q_{v}^{t o t}=q_{v s}^{t o t}\end{cases}
$$

In (2.8), the saturation value $q_{v s}^{\text {tot }}(z)$ is usually decreasing as altitude increases, so that $\mathrm{d} q_{v s}^{t o t}(z) / \mathrm{d} z<0$. As a result, one can see from (2.8) that, within a cloud, upward vertical velocity $(w>0)$ is associated with condensation $(C>0)$ and cloud formation, and, on the other hand, downward vertical velocity $(w<0)$ is associated with evaporation of cloud water $(C<0)$. Furthermore, condensation is associated with heating in $(2.3)$, and evaporation is associated with cooling. The latent heat of vaporization is a constant parameter, $L_{v}=2.5 \times 10^{6} \mathrm{~J} \mathrm{~kg}^{-1}$, and the specific heat is also a constant parameter, $c_{p}=10^{3} \mathrm{~J} \mathrm{~kg}^{-1} \mathrm{~K}^{-1}$. Note that the present version of $C$ uses instantaneous saturation adjustment, whereby the water vapour $q_{v}$ is instantaneously adjusted to maintain the saturation constraint $q_{t}=q_{v s}$, whereas this adjustment time scale is finite (but very small) in nature (e.g. Grabowski \& Morrison 2008).

The moist Boussinesq system in (2.1)-(2.8) is used as an idealized model of atmospheric dynamics with phase changes. Models like (2.1)-(2.8) have also been used for many purposes and with varying degrees of idealization (e.g. Kuo 1961; Sommeria 1976; Bretherton 1987; Cuijpers \& Duynkerke 1993; Spyksma et al. 2006; Stechmann \& Stevens 2010; Pauluis \& Schumacher 2010). Here, the moist Boussinesq system is intended to be used as an extension of dry Boussinesq models that have previously been studied in geophysical fluid dynamics (e.g. Embid \& Majda (1996), Majda (2003), Hernandez-Duenas, Smith \& Stechmann (2014) and other references described in $\S 1$ ). The moist system in (2.1)-(2.8) will reduce to the dry Boussinesq system if water vapour $q_{v}$, liquid water $q_{l}$ and condensation/evaporation $C$ are neglected.

While clouds are included in (2.1)-(2.8), they are included in their most basic form. Other aspects, such as rain and ice, are not considered here, in order to put emphasis on the basic vapour-liquid phase change, which already introduces additional non-trivial behaviour. Therefore, in comparing (2.1)-(2.8) with the set-up of Zhang et al. (2021), one distinction is that the fall velocity of rain, $V_{T}$, has been set to zero here, and it is then cloud liquid water $q_{l}$ that appears here instead of rain water $q_{r}$. Nevertheless, while many complicated cloud processes have been neglected here, the system in (2.1)-(2.8) provides the starting point for extensions that include rainfall and other complexities (e.g. Grabowski \& Smolarkiewicz 1996; Klein \& Majda 2006).

For use in the remainder of the paper, it is convenient to rewrite (2.1)-(2.8) in terms of a different set of thermodynamic variables that are conserved. In particular, define the anomalies of equivalent potential temperature, $\theta_{e}$, and total water mixing ratio, $q_{t}$, as

$$
\begin{gathered}
\theta_{e}=\theta+\frac{L_{v}}{c_{p}} q_{v}, \\
q_{t}=q_{v}+q_{l} .
\end{gathered}
$$


The evolution equations of $\theta_{e}$ and $q_{t}$ can be found by taking appropriate linear combinations of (2.3)-(2.5), and they take the form

$$
\begin{aligned}
& \frac{\mathrm{D} \theta_{e}}{\mathrm{D} t}+w \frac{\mathrm{d} \tilde{\theta}_{e}}{\mathrm{~d} z}=0, \\
& \frac{\mathrm{D} q_{t}}{\mathrm{D} t}+w \frac{\mathrm{d} \tilde{q}_{t}}{\mathrm{~d} z}=0,
\end{aligned}
$$

where the background states are defined, similar to (2.9)-(2.10), as $\tilde{\theta}_{e}(z)=\tilde{\theta}(z)+$ $\left(L_{v} / c_{p}\right) \tilde{q}_{v}(z)$ and $\tilde{q}_{t}(z)=\tilde{q}_{v}(z)+\tilde{q}_{l}(z)=\tilde{q}_{v}(z)$. The important feature of (2.11)-(2.12) is that the source term of condensation/evaporation, $C$, has been eliminated. As a result, (2.11)-(2.12) show that $\theta_{e}+\tilde{\theta}_{e}(z)$ and $q_{t}+\tilde{q}_{t}(z)$ are conserved along fluid parcel trajectories. Physically, the equivalent potential temperature is conserved because losses of water vapour $q_{v}$ are compensated by gains in heat $\theta$, as indicated by the definition in (2.9); and the total water mixing ratio, $q_{t}=q_{v}+q_{l}$, is conserved because losses of water vapour $q_{v}$ are compensated by gains in liquid water $q_{l}$.

To complete the rewriting, the buoyancy $b$ from (2.6) must also be rewritten in terms of $\theta_{e}$ and $q_{t}$. To do so, the following transformation is used:

$$
\begin{gathered}
\theta=\theta_{e}-\frac{L_{v}}{c_{p}} \min \left(q_{t}, q_{v s}\right), \\
q_{v}=\min \left(q_{t}, q_{v s}\right), \\
q_{l}=\max \left(0, q_{t}-q_{v s}\right),
\end{gathered}
$$

which is the reverse of the transformation in (2.9)-(2.10). Note that an anomalous $q_{v s}$ has been introduced, via a decomposition $q_{v s}^{\text {tot }}(z)=\tilde{q}_{v s}(z)+q_{v s}$; and the background state is chosen to be $\tilde{q}_{v s}=\tilde{q}_{t}$ so that the surplus water above saturation, $q_{t}^{t o t}-q_{v s}^{t o t}$, can be written equivalently as $q_{t}-q_{v s}$ and retains essentially the same form when written in terms of anomalies. The anomaly $q_{v s}$ will be a constant parameter here. By inserting (2.13)-(2.15) into the definition of buoyancy $b$ in (2.6), one arrives at

$$
b=g \begin{cases}\frac{\theta_{e}}{\theta_{0}}+\left(R_{v d}-\frac{L_{v}}{c_{p} \theta_{0}}\right) q_{t} & \text { if } q_{t}<q_{v s}, \\ \frac{\theta_{e}}{\theta_{0}}+\left(R_{v d}-\frac{L_{v}}{c_{p} \theta_{0}}\right) q_{v s}-\left(q_{t}-q_{v s}\right) & \text { if } q_{t} \geq q_{v s},\end{cases}
$$

which is the desired expression for buoyancy $b$ in terms of the variables $\theta_{e}$ and $q_{t}$.

To summarize the rewriting in terms of conserved variables, the original system (2.1)-(2.8) can be written in alternative form as

$$
\begin{gathered}
\frac{\mathrm{D} \boldsymbol{u}}{\mathrm{D} t}+f \hat{z} \times \boldsymbol{u}=-\nabla \phi+\hat{z} b, \\
\nabla \cdot \boldsymbol{u}=0, \\
\frac{\mathrm{D} \theta_{e}}{\mathrm{D} t}+w \frac{\mathrm{d} \tilde{\theta}_{e}}{\mathrm{~d} z}=0, \\
\frac{\mathrm{D} q_{t}}{\mathrm{D} t}+w \frac{\mathrm{d} \tilde{q}_{t}}{\mathrm{~d} z}=0,
\end{gathered}
$$

along with the definition of buoyancy $b$ from (2.16). The formulation in (2.16)-(2.20) will be used in the remainder of the paper. 


\section{Effects of clouds and phase changes on fast-wave averaging}

While the $\left(\theta, q_{v}, q_{l}\right)$ formulation in (2.1)-(2.8) is helpful for seeing the connection with a dry system, the $\left(\theta_{e}, q_{t}\right)$ formulation in (2.16)-(2.20) is helpful for another reason that is central to the goals of the present paper: for defining the slow modes of the system. The slow modes will be defined below in $\S 2.2$.

As the next two steps of the model specification, we first create a non-dimensional system, and, second, identify the small parameter $\epsilon$. To non-dimensionalize the system, reference values are chosen for all variables as in table 1 . For instance, the non-dimensional velocity $u^{*}$ is defined as $u^{*}=u / U$, by dividing the dimensional $u$ by the reference value $U$. In terms of non-dimensional quantities, the main system in (2.16)-(2.20) becomes, after dropping the superscript $*$ to ease notation,

$$
\begin{gathered}
\frac{\mathrm{D}_{h} \boldsymbol{u}_{h}}{\mathrm{D} t}+w \frac{\partial \boldsymbol{u}_{h}}{\partial z}+R o^{-1} \boldsymbol{u}_{h}^{\perp}+E u \nabla_{h} \phi=0, \\
A^{2}\left(\frac{\mathrm{D}_{h} w}{\mathrm{D} t}+w \frac{\partial w}{\partial z}\right)+E u \frac{\partial \phi}{\partial z}-\Gamma A^{2} b=0, \\
\nabla_{h} \cdot \boldsymbol{u}_{h}+\frac{\partial w}{\partial z}=0, \\
\frac{\mathrm{D}_{h} \theta_{e}}{\mathrm{D} t}+w \frac{\partial \theta_{e}}{\partial z}+F r_{1}^{-2}\left(\Gamma A^{2}\right)^{-1} w=0, \\
\frac{\mathrm{D}_{h} q_{t}}{\mathrm{D} t}+w \frac{\partial q_{t}}{\partial z}-F r_{2}^{-2}\left(\Gamma A^{2}\right)^{-1} w=0
\end{gathered}
$$

and the non-dimensional buoyancy definition,

$$
b= \begin{cases}\theta_{e}+\left(R_{v d} \frac{c_{p} \theta_{0}}{L_{v}}-1\right) q_{t} & \text { if } q_{t}<q_{v s}, \\ \theta_{e}+\left(R_{v d} \frac{c_{p} \theta_{0}}{L_{v}}-1\right) q_{v s}-\frac{c_{p} \theta_{0}}{L_{v}}\left(q_{t}-q_{v s}\right) & \text { if } q_{t} \geq q_{v s} .\end{cases}
$$

Note that the material derivative has been split into its horizontal part, $D_{h} / D t=\partial_{t}+$ $\boldsymbol{u}_{h} \cdot \nabla_{h}$, and vertical part, $w \partial_{z}$, where $\nabla_{h}=\left(\partial_{x}, \partial_{y}\right)$ is the horizontal part of the gradient operator. In the non-dimensional equations above, the non-dimensional parameters include the Rossby number $R o$, Euler number $E u$, aspect ratio $A$ and buoyancy parameter $\Gamma$, all of which are defined by analogy with the dry Boussinesq parameters (e.g. Majda 2003) and are defined here in table 2. A new parameter arises in (2.26) due to moisture: the thermodynamic parameter ratio, $c_{p} \theta_{0} / L_{v} \approx 0.1$.

Two other parameters, $F r_{1}$ and $F r_{2}$, also appear in (2.21)-(2.26), and some further explanation is required to relate them to traditional parameters of the dry Boussinesq equations. The two parameters $\mathrm{Fr}_{1}$ and $\mathrm{Fr}_{2}$ are similar to Froude numbers, and two of them appear here because the moist system involves two thermodynamic variables $\left(\theta_{e}\right.$ and $\left.q_{t}\right)$, as opposed to the dry case with one Froude number and one thermodynamic variable $(\theta)$. The definitions here are

$$
F r_{1}=\frac{U}{N_{1} H}, \quad F r_{2}=\frac{U}{N_{2} H},
$$

where $H$ is the reference height, $U$ is the reference horizontal velocity and

$$
N_{1}^{2}=\frac{g}{\theta_{0}} \frac{\mathrm{d} \tilde{\theta}_{e}}{\mathrm{~d} z}=\frac{g}{\theta_{0}} \frac{\mathrm{d}}{\mathrm{d} z}\left(\tilde{\theta}+\frac{L_{v}}{c_{p}} \tilde{q}_{v}\right),
$$




$\begin{array}{cc}\text { Variable } & \text { Reference Scale } \\ x, y & L \\ z & H \\ t & L / U \\ u(\boldsymbol{x}, t), v(\boldsymbol{x}, t) & U \\ w(\boldsymbol{x}, t) & U H / L \\ \phi(\boldsymbol{x}, t) & \Phi \\ \theta(\boldsymbol{x}, t), \theta_{e}(\boldsymbol{x}, t) & \Theta \\ q_{v}(\boldsymbol{x}, t), q_{l}(\boldsymbol{x}, t), q_{t}(\boldsymbol{x}, t), q_{v s}(z) & c_{p} \Theta / L_{v} \\ b(\boldsymbol{x}, t) & g \Theta / \theta_{o}\end{array}$

Table 1. Reference scales used for each variable to create non-dimensional equations.

$\begin{array}{ccc}\text { Symbol } & \text { Definition } & \text { Name (notes) } \\ R o & U(L f)^{-1} & \text { Rossby number } \\ E u & \Phi U^{-2} & \text { Euler number } \\ F r_{1} & U\left(N_{1} H\right)^{-1} & \text { Froude number (1) } \\ F r_{2} & U\left(N_{2} H\right)^{-1} & \text { Froude number (2) } \\ \Gamma & g \Theta \theta_{o}^{-1} L^{2}\left(U^{2} H\right)^{-1} & \text { (buoyancy parameter) } \\ A & H L^{-1} & \text { Aspect ratio }\end{array}$

Table 2. Non-dimensional parameters.

$$
N_{2}^{2}=-\frac{g}{\theta_{0}} \frac{L_{v}}{c_{p}} \frac{\mathrm{d} \tilde{q}_{t}}{\mathrm{~d} z}=-\frac{g}{\theta_{0}} \frac{L_{v}}{c_{p}} \frac{\mathrm{d} \tilde{q}_{v}}{\mathrm{~d} z}
$$

The parameters $N_{1}$ and $N_{2}$ are constants because the background gradients, $\mathrm{d} \tilde{\theta}_{e} / \mathrm{d} z$ and $\mathrm{d} \tilde{q}_{t} / \mathrm{d} z$, have been chosen to be constants here, as is typical for a Boussinesq system. The notation $N$ is used for $N_{1}$ and $N_{2}$ in analogy with the notation for the buoyancy frequency of a dry system, $N^{2}=\left(g / \theta_{0}\right) \mathrm{d} \tilde{\theta} / \mathrm{d} z$. For the moist system here, the two buoyancy frequencies are

$$
N_{u}^{2}=\frac{g}{\theta_{0}} \frac{\mathrm{d} \tilde{\theta}}{\mathrm{d} z}, \quad N_{s}^{2}=\frac{g}{\theta_{0}} \frac{\mathrm{d} \tilde{\theta}_{e}}{\mathrm{~d} z},
$$

and they involve $\theta$ and $\theta_{e}$, respectively, because, from (2.26), the non-dimensional buoyancy is $b \approx \theta_{e}-q_{t}=\theta$ in unsaturated regions and $b \approx \theta_{e}-q_{v s}=\theta_{e}-$ const. in saturated regions. These approximations neglect terms in (2.26) that are proportional to $c_{p} \theta_{0} / L_{v} \approx 0.1$, to simplify the expressions for clarity. The buoyancy frequency, or Brunt-Väisälä frequency, describes the frequency of buoyancy oscillations, and one can compute it in either the unsaturated or saturated phase (Durran \& Klemp 1982a,b). More complete expressions for the present model's $N_{u}$ and $N_{s}$ are derived by Smith \& Stechmann (2017) in their appendix without neglecting terms proportional to $c_{p} \theta_{0} / L_{v} \approx$ 0.1 . Associated with the buoyancy frequencies $N_{u}$ and $N_{s}$ are two Froude numbers,

$$
F r_{u}=\frac{U}{N_{u} H}, \quad F r_{s}=\frac{U}{N_{s} H} .
$$




\section{Effects of clouds and phase changes on fast-wave averaging}

Finally, then, by comparing (2.28) and $(2.29 a, b)$, one can see that the parameter sets $\left(N_{1}, N_{2}\right)$ and $\left(N_{u}, N_{s}\right)$ are related as

$$
N_{u}^{2}=N_{1}^{2}+N_{2}^{2}, \quad N_{s}=N_{1},
$$

and therefore the parameter sets $\left(F r_{1}, F r_{2}\right)$ and $\left(F r_{u}, F r_{s}\right)$ are related as

$$
F r_{u}^{-2}=F r_{1}^{-2}+F r_{2}^{-2}, \quad F r_{s}^{-1}=F r_{1}^{-1}
$$

As a result of these relationships, one can work with either $\left(N_{1}, N_{2}\right)$ and $\left(F r_{1}, F r_{2}\right)$ on the one hand, or with $\left(N_{u}, N_{s}\right)$ and $\left(F r_{u}, F r_{s}\right)$ on the other hand. Here, $\left(N_{1}, N_{2}\right)$ and $\left(F r_{1}, F r_{2}\right)$ will be used, since they appear directly in the equations of motion in (2.21)-(2.26) when using the variables $\theta_{e}$ and $q_{t}$.

Parameter values are chosen to represent rapid rotation (small Rossby number) and strong stratification (small Froude number). The Rossby number is defined to be the small parameter $\epsilon$,

$$
R o=\epsilon,
$$

and the other parameters are related to $R o$ in a distinguished limit as

$$
\begin{gathered}
F r_{1}=R o \frac{L}{L_{d_{1}}}=O(\epsilon), \quad F r_{2}=R o \frac{L}{L_{d_{2}}}=O(\epsilon), \quad \Gamma A^{2}=F r_{1}^{-1}=O\left(\epsilon^{-1}\right), \\
E u^{-1}=R o=\epsilon, \quad \frac{c_{p} \theta_{0}}{L_{v}}=C_{c l} R o=O(\epsilon),
\end{gathered}
$$

where $L_{d_{1}}$ and $L_{d_{2}}$ are similar to Rossby radii of deformation and are defined as

$$
L_{d_{1}}=\frac{N_{1} H}{f}, \quad L_{d_{2}}=\frac{N_{2} H}{f} .
$$

With these parameter relationships, the non-dimensional equations from (2.21)-(2.26) become

$$
\begin{gathered}
\frac{\mathrm{D}_{h} \boldsymbol{u}_{h}}{\mathrm{D} t}+w \frac{\partial \boldsymbol{u}_{h}}{\partial z}+\epsilon^{-1} \boldsymbol{u}_{h}^{\perp}+\epsilon^{-1} \nabla_{h} \phi=0, \\
A^{2}\left(\frac{\mathrm{D}_{h} w}{\mathrm{D} t}+w \frac{\partial w}{\partial z}\right)+\epsilon^{-1} \frac{\partial \phi}{\partial z}-\epsilon^{-1} \frac{L_{d_{1}}}{L} b=0, \\
\nabla_{h} \cdot \boldsymbol{u}_{h}+\frac{\partial w}{\partial z}=0, \\
\frac{\mathrm{D}_{h} \theta_{e}}{\mathrm{D} t}+w \frac{\partial \theta_{e}}{\partial z}+\epsilon^{-1} \frac{L_{d_{1}}}{L} w=0, \\
\frac{\mathrm{D}_{h} q_{t}}{\mathrm{D} t}+w \frac{\partial q_{t}}{\partial z}-\epsilon^{-1} \frac{L_{d_{2}}}{L} \frac{L_{d_{2}}}{L_{d_{1}}} w=0
\end{gathered}
$$

and the non-dimensional buoyancy definition,

$$
b= \begin{cases}\theta_{e}+\left(\epsilon R_{v d} C_{c l}-1\right) q_{t} & \text { if } q_{t}<q_{v s}, \\ \theta_{e}+\left(\epsilon R_{v d} C_{c l}-1\right) q_{v s}-\epsilon C_{c l}\left(q_{t}-q_{v s}\right) & \text { if } q_{t} \geq q_{v s} .\end{cases}
$$

In arriving at (2.37)-(2.42), the scaling scenario is similar to dry fast-wave averaging (e.g. Embid \& Majda 1996), with extensions to the present case with moisture, following 


\section{Y. Zhang, L.M. Smith and S.N. Stechmann}

Smith \& Stechmann (2017), and discussed further below. While one could proceed with (2.37)-(2.42), the analysis would be complicated by the many $O(1)$ constants that appear: $A, L / L_{d_{1}}, L / L_{d_{2}}, R_{v d}$ and $C_{c l}$. To simplify the notation in what follows, these $O(1)$ constants will be set equal to unity, which leads to

$$
\begin{gathered}
\frac{\mathrm{D}_{h} \boldsymbol{u}_{h}}{\mathrm{D} t}+w \frac{\partial \boldsymbol{u}_{h}}{\partial z}+\epsilon^{-1} \boldsymbol{u}_{h}^{\perp}+\epsilon^{-1} \nabla_{h} \phi=0, \\
\frac{\mathrm{D}_{h} w}{\mathrm{D} t}+w \frac{\partial w}{\partial z}+\epsilon^{-1} \frac{\partial \phi}{\partial z}-\epsilon^{-1} b=0, \\
\nabla_{h} \cdot \boldsymbol{u}_{h}+\frac{\partial w}{\partial z}=0 \\
\frac{\mathrm{D}_{h} \theta_{e}}{\mathrm{D} t}+w \frac{\partial \theta_{e}}{\partial z}+\epsilon^{-1} w=0 \\
\frac{\mathrm{D}_{h} q_{t}}{\mathrm{D} t}+w \frac{\partial q_{t}}{\partial z}-\epsilon^{-1} w=0
\end{gathered}
$$

and the non-dimensional buoyancy definition,

$$
b= \begin{cases}\theta_{e}+(\epsilon-1) q_{t} & \text { if } q_{t}<q_{v s} \\ \theta_{e}+(\epsilon-1) q_{v s}-\epsilon\left(q_{t}-q_{v s}\right) & \text { if } q_{t} \geq q_{v s} .\end{cases}
$$

where each term in (2.43)-(2.48) now has a coefficient that is either unity or $\epsilon$. This completes the specification of the model, in non-dimensional units, in (2.43)-(2.48), for use in the remainder of the manuscript.

To provide some additional physical context, we describe how the scaling regime used here is reminiscent of the midlatitude atmosphere on synoptic scales, if a value of $\epsilon \approx 0.1$ is used (e.g. Majda 2003; Vallis 2006; Smith \& Stechmann 2017). For instance, one can arrive at $R o, F r_{1}, F_{2}$ values of approximately 0.1 in the following way. For $f=10^{-4} \mathrm{~s}^{-1}$, $L=10^{6} \mathrm{~m}$ and $U=10 \mathrm{~m} \mathrm{~s}^{-1}$, the Rossby number is $R o=0.1$; and for $\mathrm{d} \tilde{\theta} / \mathrm{d} z=3 \mathrm{~K} \mathrm{~km}^{-1}$, $d \tilde{q}_{v} / d z=-0.6 \mathrm{~g} \mathrm{~kg}^{-1} \mathrm{~km}^{-1}$ and $H=10^{4} \mathrm{~m}$, one has $N_{1}^{2} \approx N_{2}^{2}$ and $F r_{1} \approx F r_{2} \approx 0.14$. In this case, the buoyancy frequencies are related as $N_{u}^{2} \approx 2 N_{s}^{2}$, consistent with a moist (or saturated) buoyancy frequency that is lower frequency than the dry (or unsaturated) buoyancy frequency. Hence the scaling choice of $F r_{1}=F r_{2}$ is physically realistic in this sense. One scaling choice that deviates slightly from established physical values is the choice of $R_{v d}=1$, where $R_{v d}$ is a parameter that is related to the gas constants for water vapour and dry air, as described above, and its value in reality is 0.61 ; nevertheless, this term does not arise at leading order in (2.42) and is not a central aspect of the analysis here. Also, the aspect ratio has been chosen to be $A=1$ here, which differs from typical values for the midlatitude atmosphere on synoptic scales (e.g. Vallis 2006; Smith \& Stechmann 2017), but is consistent with earlier studies of fast-wave averaging in the dry case (e.g. Embid \& Majda 1996; Majda 2003). Indeed, in summarizing the parameter choices, the present study is an investigation of fast-wave averaging, and it uses parameter values that are consistent with and motivated by the midlatitude atmosphere on synoptic scales, but it is an idealized set-up, as in earlier fast-wave averaging studies (e.g. Embid \& Majda 1996; Majda 2003). As such, the present paper will consider a variety of different $\epsilon$ values as $\epsilon$ tends toward zero. 


\section{Effects of clouds and phase changes on fast-wave averaging}

For later use, we note that the buoyancy $b$ in (2.48) can be written in an alternative form as

$$
b=H_{u} b_{u}+H_{s} b_{s},
$$

where $H_{u}$ and $H_{s}$ are Heaviside functions that indicate the unsaturated and saturated phases, respectively:

$$
H_{u}=\left\{\begin{array}{ll}
1 & \text { for } q_{t}<q_{v s}, \\
0 & \text { for } q_{t} \geq q_{v s},
\end{array} \quad \text { and } \quad H_{s}=1-H_{u} .\right.
$$

In (2.49), the expressions for the unsaturated buoyancy $b_{u}$ and the saturated buoyancy $b_{s}$ are given by

$$
b_{u}=\theta_{e}+(\epsilon-1) q_{t}, \quad b_{s}=\theta_{e}+(\epsilon-1) q_{v s}-\epsilon\left(q_{t}-q_{v s}\right),
$$

which follow from comparison with the earlier expression for $b$ in (2.48). By using the Heaviside functions, the expression in (2.49) can be used to describe the buoyancy succinctly, and the Heaviside functions provide a clear indication that the form of the buoyancy will change in different phases.

\subsection{Slow variables}

The premise of fast-wave averaging depends on separation of slowly varying and fast-wave components of the system. On the one hand, when fast waves evolve in time, they are influenced by the $\epsilon^{-1}$ terms in the moist Boussinesq system in (2.43)-(2.48). Physically, the $\epsilon^{-1}$ terms represent the effects of rapid rotation and strong stratification, which can cause fast-wave oscillations. On the other hand, slow variables will have evolution equations that are not influenced by the $\epsilon^{-1}$ terms.

Keeping these features in mind, we here present an algebraic construction of the slow variables (a derivation using operator analysis may be found in Zhang et al. (2021)). The construction draws upon foundations from previous literature regarding dry and moist dynamics, in particular, to identify potential vorticity variables (see e.g. Majda (2003), Marquet (2014), Wetzel et al. (2020) and references therein). In addition, for the moist system described in $\S 2.1$, a second slow variable has been found and named $M$ (Smith \& Stechmann 2017; Wetzel et al. 2019b, 2020).

First, to define the slow variable $M$, we follow the intuition mentioned above: the goal is to construct a new quantity whose evolution equation does not involve any $\epsilon^{-1}$ terms. We look into the evolution equations for $\theta_{e}$ and $q_{t}$ in (2.46)-(2.47). In particular, while the $\theta_{e}$ evolution in (2.46) involves an $\epsilon^{-1} w$ term, and while the $q_{t}$ evolution in (2.47) involves a $-\epsilon^{-1} w$ term, if we take their sum we can eliminate the $\epsilon^{-1}$ terms. Therefore, it is straightforward to eliminate the $\epsilon^{-1} w$ terms from the $\theta_{e}$ and $q_{t}$ equations using the linear combination

$$
M=q_{t}+\theta_{e},
$$

which obeys the evolution equation of

$$
\frac{\mathrm{D} M}{\mathrm{D} t}=0
$$

Physically, the quantity $M^{2}$ also has an interpretation as a moist latent energy that is released upon change of phase (Marsico, Smith \& Stechmann 2019).

Because (2.53) involves no explicit $\epsilon^{-1}$ terms, $M$ is referred to as a slow variable. 


\section{Y. Zhang, L.M. Smith and S.N. Stechmann}

Note that $M$ can be shown to be slowly evolving only in certain settings where the notions of slow and fast can be appropriately defined. One could look to either the linearized or nonlinear setting. In a linearized setting, if (2.53) is linearized about a resting base state with $\boldsymbol{u}=0$, then the resulting linearized equation is $\partial M / \partial t=0$, so that $M$ is slowly varying and in fact does not change in time at all. This is true in the linearized setting for any value of $\epsilon$, large or small. In a nonlinear setting, one can also show that $M$ is slowly varying, if one considers the setting of a small $\epsilon$ limit, so that the slow time scale is defined to be time of $O(1)$ duration, and the fast time scales are of $O(\epsilon)$ duration. The case of balanced initial conditions was considered by Smith \& Stechmann (2017), and the case of unbalanced initial conditions was considered by Zhang et al. (2021) as well as in the present paper, further below. These same statements also hold true for the other slow variable, potential vorticity.

Second, following a similar procedure, one can look for ways to cancel the $\epsilon^{-1}$ terms in the momentum evolution, (2.43), to define a slow variable called potential vorticity. By applying a horizontal curl $\left(\nabla_{h} \times\right)$ to the momentum evolution in (2.43), the $\epsilon^{-1}$ term from the pressure gradient is eliminated, and the resulting equation is

$$
\frac{\partial}{\partial t} \nabla_{h} \times \boldsymbol{u}_{h}+\nabla_{h} \times\left(\boldsymbol{u}_{h} \cdot \nabla_{h} \boldsymbol{u}_{h}+w \frac{\partial \boldsymbol{u}_{h}}{\partial z}\right)+\epsilon^{-1} \nabla_{h} \cdot \boldsymbol{u}_{h}=0 .
$$

Then, one can see that the remaining $\epsilon^{-1}$ term in (2.54) involves $\nabla_{h} \cdot \boldsymbol{u}_{h}$; to eliminate it, one can form the potential vorticity variable defined as

$$
P V_{e}=\xi+\frac{\partial \theta_{e}}{\partial z}, \quad \text { where } \xi=\nabla_{h} \times \boldsymbol{u}_{h},
$$

i.e. $\xi$ is the vertical component of the total vorticity $\nabla \times \boldsymbol{u}$. To find the evolution equation for $P V_{e}$, apply $\partial_{z}$ to (2.46) and add the result to (2.54) to arrive at

$$
\frac{\partial P V_{e}}{\partial t}+\frac{\partial\left(\boldsymbol{u} \cdot \nabla \theta_{e}\right)}{\partial z}+N L_{\xi}=0
$$

where

$$
N L_{\xi}=\nabla_{h} \times\left(\boldsymbol{u}_{h} \cdot \nabla_{h} \boldsymbol{u}_{h}+w \frac{\partial \boldsymbol{u}_{h}}{\partial z}\right)=\boldsymbol{u} \cdot \nabla \xi+\xi\left(u_{x}+v_{y}\right)+\left(w_{x} v_{z}-w_{y} u_{z}\right) .
$$

Then the material derivative of $P V_{e}$ is given by

$$
\frac{\mathrm{D} P V_{e}}{\mathrm{D} t}=-\left(\boldsymbol{u}_{z} \cdot \nabla \theta_{e}\right)-\xi\left(u_{x}+v_{y}\right)-\left(w_{x} v_{z}-w_{y} u_{z}\right) .
$$

On the right-hand side of this equation, the latter two terms represent vortex stretching, as can be seen from their origins from the vorticity equation in (2.54) and from (2.57); and the first term on the right-hand side arises from advection of $\theta_{e}$. The main property of interest here is that, since this $P V_{e}$ evolution equation contains no explicit $\epsilon^{-1}$ terms, $P V_{e}$ is referred to as a slow variable.

Note that a variety of different moist $P V$ variables have been proposed and used for various purposes (e.g. Bennetts \& Hoskins 1979; Emanuel 1979; Cao \& Cho 1995; Schubert et al. 2001; Marquet 2014; Smith \& Stechmann 2017). Different $P V$ variables can have different properties, and, in fact, some common choices of moist $P V$ are not slowly varying. For instance, a moist $P V$ variable based on potential temperature, $\theta$, or on virtual potential temperature, $\theta_{v}$, is not slowly varying (Wetzel et al. 2020). The $P V_{e}$ 

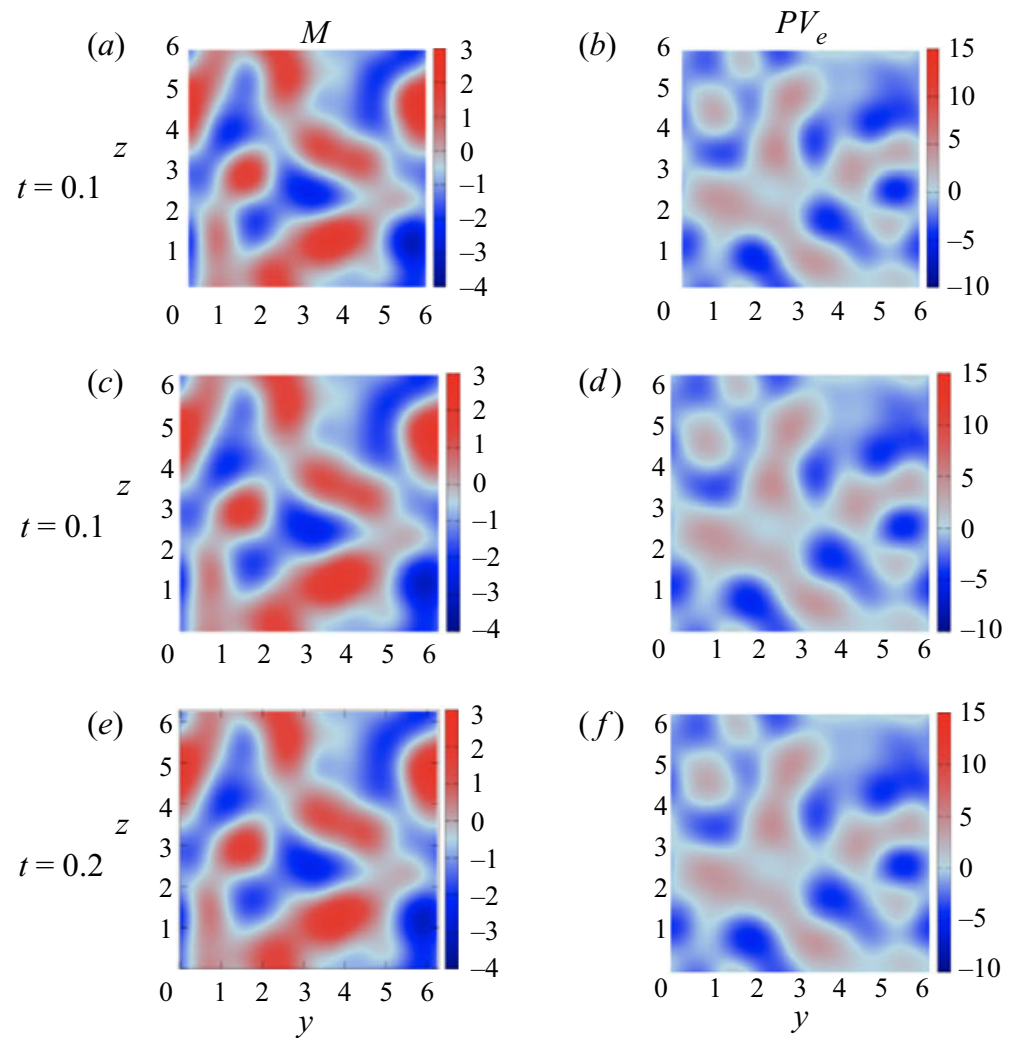

Figure 1. Evolution of slow modes $M(a, c, e)$ and $P V_{e}(b, d, f)$ on short times of $t=O(\epsilon) \sim 10^{-1}$, starting from large-scale random initial conditions at $t=0$. The two-dimensional (2-D) slices are taken with $x=\pi$ held fixed. One can see that the slow modes $\left(M, P V_{e}\right)$ change very little over short times $t=O(\epsilon)$.

variable, based on $\theta_{e}$, is one definition of $P V$ that is slowly varying, and it is therefore well-suited for the present study.

To visualize that $\left(M, P V_{e}\right)$ are slowly varying, figure 1 shows the short-time evolution of $M(a, c, e)$ and $P V_{e}(b, d, f)$. The system (2.43)-(2.48) evolves in a triply periodic domain from random, large-scale initial conditions with $\epsilon=O(0.1)$ (see $\S 3.1)$. To the eye, it is apparent that $\left(M, P V_{e}\right)$ are almost invariant for times $t=O(\epsilon) \sim 10^{-1}$. We note that (2.43)-(2.48) have been non-dimensionalized using the advective time scale, such that one expects variation of the slow variable on the times $t=O(1)$.

While $P V_{e}$ and $M$ represent the slow components, additional variables are needed to represent the fast components of the system, and thereby to completely specify the entire dynamics. Formally, we may divide the phase space into $\left(M, P V_{e}\right)$ and the wave complement $\left(W_{1}, W_{2}, u_{m}, v_{m}\right)$, where $\left(W_{1}, W_{2}\right)$ are analogous to dry inertia-gravity waves involving the vertical velocity $w$, and $\left(u_{m}, v_{m}\right)$ are the horizontal mean velocities corresponding to inertial waves (Gill 1982; Remmel \& Smith 2009; Remmel 2010; Hernandez-Duenas et al. 2014).

Hence, we will make a change of variables to utilize the two quantities $P V_{e}$ and $M$ that characterize the slowly varying subspace.

The slow variables, $M$ and $P V_{e}$, will be the central focus of the remainder of the paper. They are analogous to the dry $P V$ that is the central focus of dry fast-wave averaging (e.g. Embid \& Majda 1996; Majda 2003). In the dry case, in the limit of small $\epsilon$, 


\section{Y. Zhang, L.M. Smith and S.N. Stechmann}

the $P V$ variable has a remarkable property: it obeys a nonlinear evolution that is decoupled from the evolution of the fast waves. Here, in the moist case, our goal is to investigate the evolution of the slow variables $M$ and $P V_{e}$, and to see whether they have a decoupled evolution, or whether phase changes bring about coupling with waves.

\subsection{Abstract form of the equations}

Many systems related to dry atmospheric dynamics may be written in abstract form as

$$
\frac{\partial \boldsymbol{v}}{\partial t}+\mathcal{L}(\boldsymbol{v})+\mathscr{B}(\boldsymbol{v}, \boldsymbol{v})=0
$$

where $v$ is the state vector, the linear operator $\mathcal{L}$ includes the effects of rotation and buoyancy, and $\mathcal{B}$ is bilinear (Majda 2003). On the other hand, the buoyancy changes its functional form across phase interfaces in dynamics with phase changes of water. In the latter case, (2.59) must be rewritten as

$$
\frac{\partial \boldsymbol{v}}{\partial t}+H_{u}(\boldsymbol{v}) \mathcal{L}_{u}(\boldsymbol{v})+H_{s}(\boldsymbol{v}) \mathcal{L}_{s}(\boldsymbol{v})+\mathscr{B}(\boldsymbol{v}, \boldsymbol{v})=0 .
$$

This is the abstract form of the model in (2.43)-(2.48), where the linear term $\mathcal{L}(\boldsymbol{v})$ has been replaced by $H_{u}(\boldsymbol{v}) \mathcal{L}_{u}(\boldsymbol{v})+H_{s}(\boldsymbol{v}) \mathcal{L}_{s}(\boldsymbol{v})$ to account for the effect of phase changes as described in (2.49)-(2.51a,b). Each of the linear operators, $\mathcal{L}_{u}$ and $\mathcal{L}_{s}$, is by itself a constant-coefficient operator. However, their prefactors, $H_{u}(\boldsymbol{v})$ and $H_{S}(\boldsymbol{v})$ depend on $\boldsymbol{v}$ such that $H_{u}(\boldsymbol{v}) \mathcal{L}_{u}(\boldsymbol{v})+H_{S}(\boldsymbol{v}) \mathcal{L}_{S}(\boldsymbol{v})$ is nonlinear.

Here, we assume existence of (2.43)-(2.48), and, consequently, the solution $\boldsymbol{v}^{\epsilon}(\boldsymbol{x}, t)$ is assumed to be known for each value of $\epsilon$. Then for known $\boldsymbol{v}^{\epsilon}(\boldsymbol{x}, t)$, the goal of fast-wave averaging is to assess the coupling between the slow and fast components of the flow. From this perspective, we treat the Heaviside functions $\left(H_{u}, H_{s}\right)$ as given functions of $(\boldsymbol{x}, t)$ at the stages of the fast-wave-averaging analysis. Thus the abstract formulation is, $a$ posteriori, restored to its original form (2.59) where $\mathcal{L}=H_{u}(\boldsymbol{x}, t) \mathcal{L}_{u}+H_{s}(\boldsymbol{x}, t) \mathcal{L}_{s}$. As a result, many of the techniques from prior fast-wave-averaging studies can be applied here to the case with phase changes.

\subsection{Fast-wave averaging}

Fast waves arise in (2.59) when the operator $\mathcal{L}$ has a large $O\left(\epsilon^{-1}\right)$ contribution for small $\epsilon \rightarrow 0$. In this case, the operator $\mathcal{L}$ may be decomposed as $\mathcal{L}=\epsilon^{-1} \mathcal{L}_{*}+\mathcal{L}_{0}$, so that (2.59) may be rewritten as

$$
\frac{\partial \boldsymbol{v}}{\partial t}+\epsilon^{-1} \mathcal{L}_{*}(\boldsymbol{v})+\mathcal{L}_{0}(\boldsymbol{v})+\mathcal{B}(\boldsymbol{v}, \boldsymbol{v})=0, \quad \boldsymbol{v}(x, 0)=\bar{v}(\boldsymbol{x})
$$

where the dominant terms are identified by the multiplier $\epsilon^{-1}$ and $\bar{v}(\boldsymbol{x})$ is the initial state. As discussed, we treat phase boundaries $\left(H_{u}, H_{S}\right)$ as known functions of $(\boldsymbol{x}, t)$ for the fast-wave averaging analysis, and proceed to analyse (2.61) together with

$$
\mathcal{L}=H_{u} \mathcal{L}_{u}+H_{s} \mathcal{L}_{s}=\epsilon^{-1} \mathcal{L}_{*}+\mathcal{L}_{0},
$$

for given $\left(H_{u}, H_{s}\right)$.

The idea of fast-wave averaging is to use a two-time expansion

$$
\boldsymbol{v}^{\epsilon}(\boldsymbol{x}, t)=\boldsymbol{v}^{0}(\boldsymbol{x}, t, \tau)+\epsilon \boldsymbol{v}^{1}(\boldsymbol{x}, t, \tau)+\cdots, \quad \epsilon \rightarrow 0,
$$

where $\tau=t / \epsilon$ is the fast time scale. Inserting (2.63) into (2.61), and collecting terms order-by-order in $\epsilon$, leads to a series of initial value problems whose solutions may 


\section{Effects of clouds and phase changes on fast-wave averaging}

be found explicitly in terms of the initial state $\bar{v}(\boldsymbol{x})$. The condition to suppress secular growth of $v^{1}$ is referred to as the fast-wave-averaged equation (Majda 2003). Since phase interfaces are determined by the complete (thermo)dynamics, they have a fast component (dependence on $\tau$ in the two-time expansion). Therefore, a main new element of the formulation is the $\tau$ dependence in the linear operator $\mathcal{L}_{*}(t, \tau)$. In the limit $\epsilon \rightarrow 0$, $\tau=t / \epsilon \rightarrow \infty$ with $t=O(1)$, the fast-wave-averaged equation is thus given by

$$
\begin{gathered}
\frac{\partial \bar{v}(\boldsymbol{x}, t)}{\partial t}=\lim _{\tau \rightarrow \infty} \frac{1}{\tau} \int_{0}^{\tau}\left\{\left(\int_{0}^{s} \frac{\partial \mathcal{L}_{*}\left(t, s^{\prime}\right)}{\partial t} \mathrm{~d} s^{\prime}\right) \bar{v}-\exp \left(\int_{0}^{s} \mathcal{L}_{*}\left(t, s^{\prime}\right) \mathrm{d} s^{\prime}\right)\right. \\
\times\left[\mathcal{L}_{0}(t, s)\left(\exp \left(-\int_{0}^{s} \mathcal{L}_{*}\left(t, s^{\prime}\right) \mathrm{d} s^{\prime}\right) \bar{v}\right)\right. \\
\left.\left.+\mathcal{B}\left(\exp \left(-\int_{0}^{s} \mathcal{L}_{*}\left(t, s^{\prime}\right) \mathrm{d} s^{\prime}\right) \bar{v}, \exp \left(-\int_{0}^{s} \mathcal{L}_{*}\left(t, s^{\prime}\right) \mathrm{d} s^{\prime}\right) \bar{v}\right)\right]\right\} \mathrm{d} s .
\end{gathered}
$$

Another significant difference from the dry analysis is that the calculations to assess coupling on the right-hand side of (2.64) must be performed in physical space, rather than relying on Fourier analysis, owing to the piecewise nature of the buoyancy operator.

For compactness, most of the details to derive (2.64), and its projection onto $\left(M, P V_{e}\right)$ (see (2.72) and (2.73)), are presented elsewhere (Zhang et al. 2021). A sketch of the steps to arrive at (2.64) is given in Appendix A. In addition, the terms appearing in the final result (2.72)-(2.73) can be understood by comparison with the structure of $\left(M, P V_{e}\right)$ equations (2.53) and (2.56), as will be further explained at the end of the next section, $\S 2.5$.

\subsection{Fast-wave-averaged equations for $M$ and $P V_{e}$}

To focus on the evolution of the slow variables $M$ and $P V_{e}$, and possible decoupling of their evolution from fast oscillations, we may project (2.64) onto the first two components of $\bar{v}=\left.\left(M, P V_{e}, W_{1}, W_{2}, u_{m}, v_{m}\right)\right|_{\tau=0}$. To this end, let us separate slow and fast components using the following definitions:

$$
\bar{v}(x, t)=\bar{v}_{\left(M, P V_{e}\right)}(x, t)+\bar{v}_{(W)}(\boldsymbol{x}, t),
$$

where

$$
\bar{v}_{\left(M, P V_{e}\right)}(\boldsymbol{x}, t)=\left(\begin{array}{c}
M(\boldsymbol{x}, t) \\
P V_{e}(\boldsymbol{x}, t) \\
0 \\
0 \\
0 \\
0
\end{array}\right), \quad \bar{v}_{(W)}(\boldsymbol{x}, t)=\left(\begin{array}{c}
0 \\
0 \\
W_{1}(\boldsymbol{x}, t, 0) \\
W_{2}(\boldsymbol{x}, t, 0) \\
u_{m}(\boldsymbol{x}, t, 0) \\
v_{m}(\boldsymbol{x}, t, 0)
\end{array}\right) .
$$

The nomenclature 'slow' and 'fast' follows naturally from $\mathcal{L}_{*} \bar{v}_{\left(M, P V_{e}\right)}=0$ while $\mathcal{L}_{*} \bar{v}_{(W)} \neq 0$. For ease of notation in the following evolution equations, we introduce the quantity

$$
\bar{v}_{\left(W^{\prime}\right)}=\exp \left(\int_{0}^{s} \mathcal{L}_{*}\left(t, s^{\prime}\right) \mathrm{d} s^{\prime}\right) \bar{v}_{(W)} .
$$

The fast-wave-averaged equation for the slow variable $M$ may be written as

$$
\frac{\partial M(\boldsymbol{x}, t)}{\partial t}=-\lim _{\tau \rightarrow \infty}\left(\frac{1}{\tau} \int_{0}^{\tau} \boldsymbol{u}_{\left(M, P V_{e}\right)}(\boldsymbol{x}, t, s) \mathrm{d} s+\frac{1}{\tau} \int_{0}^{\tau} \boldsymbol{u}_{\left(W^{\prime}\right)}(\boldsymbol{x}, t, s) \mathrm{d} s\right) \cdot \nabla M(\boldsymbol{x}, t),
$$




\section{Y. Zhang, L.M. Smith and S.N. Stechmann}

where $\nabla M$ does not depend on $\tau$, and thus may be taken outside of the integrals. The velocity $\boldsymbol{u}_{\left(M, P V_{e}\right)}$ associated with $\left(M, P V_{e}\right)$ is found from $\left(M, P V_{e}\right)$ inversion,

$$
\nabla_{h}^{2} \psi+\frac{\partial}{\partial z}\left[H_{u}\left(\frac{1}{2} \frac{\partial \psi}{\partial z}+\frac{1}{2} M\right)\right]+\frac{\partial}{\partial z}\left[H_{s}\left(\frac{\partial \psi}{\partial z}+q v\right)\right]=P V_{e},
$$

where

$$
\boldsymbol{u}_{\left(M, P V_{e}\right)}=\left(-\frac{\partial \psi}{\partial y}, \frac{\partial \psi}{\partial x}, 0\right), \quad \theta_{e\left(M, P V_{e}\right)}=\frac{1}{2} H_{u}\left(\frac{\partial \psi}{\partial z}+M\right)+H_{s}\left(\frac{\partial \psi}{\partial z}+q v s\right)
$$

Relation (2.69) is one of the formulae to prescribe the transformation between $\left(u, v, w, \theta_{e}, q_{t}\right)$ and $\left(M, P V_{e}, W_{1}, W_{2}, u_{m}, v_{m}\right)$, with the wave complement set equal to zero, and is the analogue of $P V$ inversion in the dry dynamics (Smith \& Stechmann 2017; Wetzel et al. 2019b, 2020). Once $\boldsymbol{u}_{\left(M, P V_{e}\right)}$ has been found from $\left(M, P V_{e}\right)$ inversion, one may compute $\boldsymbol{u}_{(W)}=\boldsymbol{u}-\boldsymbol{u}_{\left(M, P V_{e}\right)}$, and then use (2.67) to compute $\boldsymbol{u}_{\left(W^{\prime}\right)} \sim \boldsymbol{u}_{(W)}, \epsilon \rightarrow 0$.

To aid in the interpretation of (2.68), we use the notation $\langle f\rangle$ to define the time average of any function $f(\boldsymbol{x}, t, \tau)$, as follows:

$$
\langle f\rangle(\boldsymbol{x}, t)=\lim _{\tau \rightarrow \infty} \frac{1}{\tau} \int_{0}^{\tau} f(\boldsymbol{x}, t, s) \mathrm{d} s .
$$

Using the bracket \langle\rangle notation, the $M$-evolution equation (2.68) becomes

$$
\frac{\partial M(\boldsymbol{x}, t)}{\partial t}=-\left\langle\boldsymbol{u}_{\left(M, P V_{e}\right)}\right\rangle(\boldsymbol{x}, t) \cdot \nabla M(\boldsymbol{x}, t)-\left\langle\boldsymbol{u}_{\left(W^{\prime}\right)}\right\rangle(\boldsymbol{x}, t) \cdot \nabla M(\boldsymbol{x}, t),
$$

in which there are two different contributions involving time-averaged velocity fields, $\left\langle\boldsymbol{u}_{\left(M, P V_{e}\right)}\right\rangle$ and $\left\langle\boldsymbol{u}_{\left(W^{\prime}\right)}\right\rangle$.

One of the significant differences between the dry case and phase-change case can now be seen. In particular, in the dry case, a dichotomy exists: the vortical eigenmode is slow, and the wave eigenmode is fast. In the phase-change case, this clean dichotomy no longer exists. As one indication of this, notice that the Heaviside functions $H_{u}$ and $H_{s}$ in (2.69) are influenced by waves. Therefore, the PDE in (2.69) has parameters $\left(H_{u}\right.$ and $\left.H_{S}\right)$ that include fast-wave oscillations, and the solution $\psi$ will also be influenced by fast wave oscillations. Similarly, since the velocity $\boldsymbol{u}_{\left(M, P V_{e}\right)}$ is calculated from $\psi$ via $(2.70 a, b)$, it too is influenced by fast-wave oscillations. Interestingly, though, the quantities $P V_{e}$ and $M$ are indeed purely slow quantities, as indicated above, even though other quantities $\left(\psi, \boldsymbol{u}_{\left(M, P V_{e}\right)}\right.$, etc.) that are derived from $P V_{e}$ and $M$ are not purely slow quantities. Hence, in the phase-change case, one must analyse the average $\left\langle\boldsymbol{u}_{\left(M, P V_{e}\right)}\right\rangle$ as $\tau \rightarrow \infty$ in order to know the evolution of the slow variable $M$ in (2.72). On the other hand, in the dry case (or purely saturated case without phase changes), one has $\left\langle\boldsymbol{u}_{\left(M, P V_{e}\right)}\right\rangle=\boldsymbol{u}_{\left(M, P V_{e}\right)}$.

Using inversion formulae, and similar to the decomposition $\boldsymbol{u}=\boldsymbol{u}_{\left(M, P V_{e}\right)}+\boldsymbol{u}_{(W)}$, all primary variables $\left(u, v, w, \theta_{e}, q_{t}\right)$ may be decomposed into a component associated with $\left(M, P V_{e}\right)$ and a component associated with the waves $(W)$. Although there is $\tau$ dependence remaining in the $\left(M, P V_{e}\right)$ part, for the sake of simplicity and continuity with previous literature, we proceed to adopt the language convention that slow refers to $(\cdot)_{\left(M, P V_{e}\right)}$, while fast refers to $(\cdot)_{(W)}$. Using that convention, slow-slow (fast-fast) nonlinear interactions involve products between two quantities associated with $\left(M, P V_{e}\right)(W)$. Mixed slow-fast and fast-slowquadratic terms involve one of each type. Thus the first (second) term on the right-hand side of (2.72) is a slow-slow (fast-slow) term.

Figure 2 shows the short time evolution of the total water $q_{t}$ (panels $(a, d, g)$ ), along with the slow part $q_{t\left(M, P V_{e}\right)}$ and the fast part $q_{t(W)}$. The simulation is the same as in figure 1 

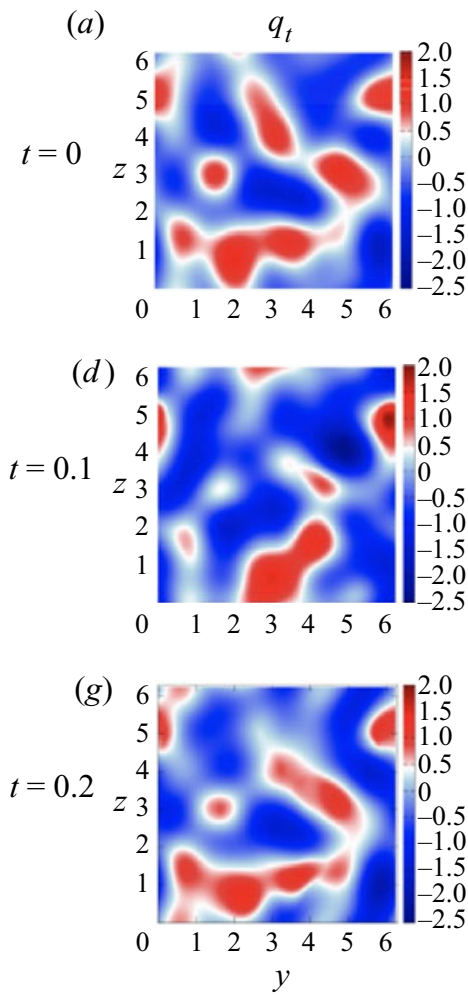
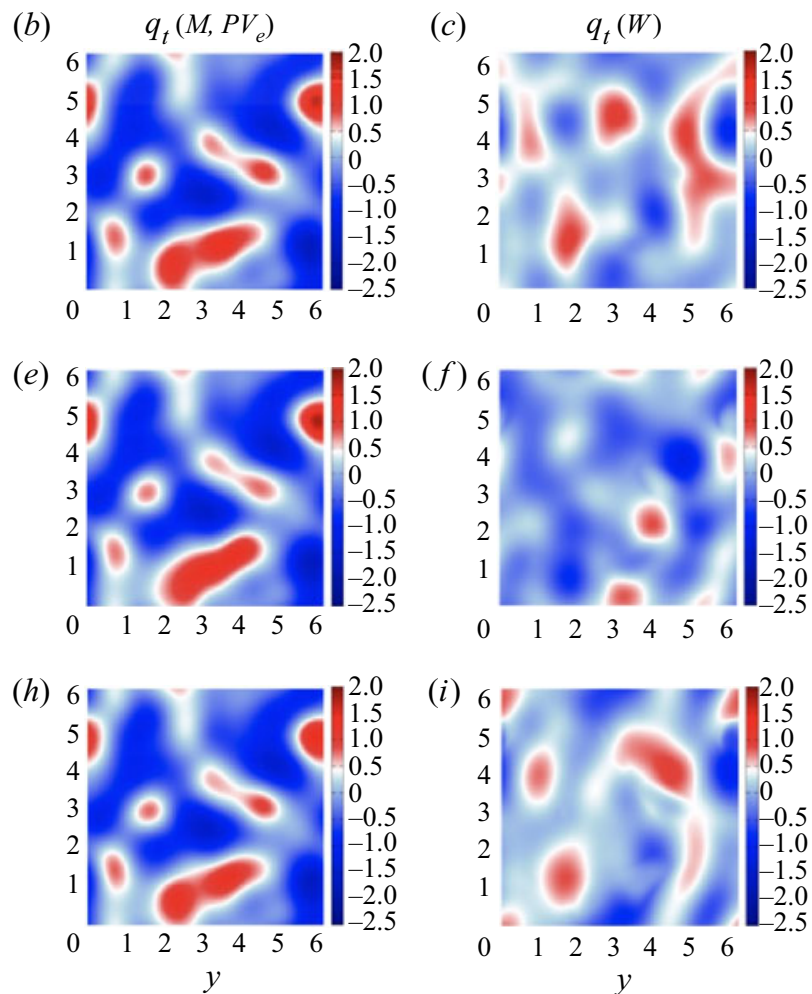

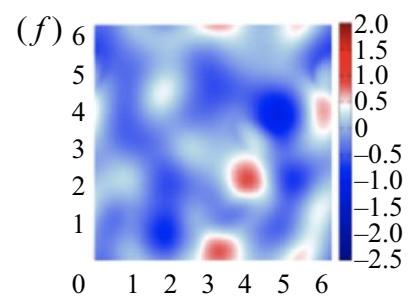

(i)

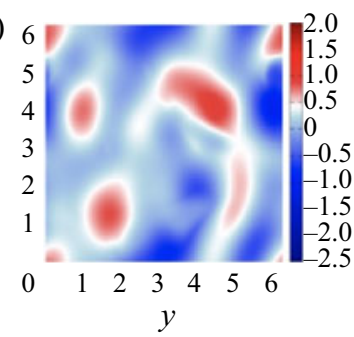

Figure 2. Short-time evolution of total water $q_{t}=q_{t\left(M, P V_{e}\right)}+q_{t(W)}(a, d, g)$, slow water $q_{t\left(M, P V_{e}\right)}(b, e, h)$ and fast water $q_{t(W)}(c, f, i)$. The simulation is the same as in figure 1 , and 2-D slices have $x=\pi$ held fixed. The saturation threshold $q_{v s}=0.5$, such that red corresponds to liquid water and blue corresponds to water vapour. The slow component $q_{t\left(M, P V_{e}\right)}$ changes very little on times $t=O(\epsilon)$, while the fast component $q_{t(W)}$ shows wave-like behaviour with $O(1)$ amplitude variation at fixed $x$ over times $t=O(\epsilon)$.

(decay from large-scale initial conditions with $\epsilon=O(0.1)$ ), and short times correspond to $t=O(\epsilon) \sim 10^{-1}$. Despite the $\tau$ dependence of $q_{t\left(M, P V_{e}\right)}$, it changes very little at large scales, while the variation of the unbalanced water $q_{t(W)}$ is much more significant. Our goal is to assess how the slow flow components are influenced by the fast components on $O(1)$ time scales, and in particular we use fast-wave averaging to study how the slow modes $\left(M, P V_{e}\right)$ are coupled to waves by analysing the nonlinear terms on the right-hand side of the $M$-equation (2.72).

To complete the picture, the fast-wave-averaged equation for $P V_{e}$ has also been derived from (2.64), and may be written as

$$
\begin{aligned}
-\frac{\partial P V_{e}(\boldsymbol{x}, t)}{\partial t}= & \left\langle\boldsymbol{u}_{\left(M, P V_{e}\right)}\right\rangle(\boldsymbol{x}, t) \cdot \nabla P V_{e}(\boldsymbol{x}, t)+\left\langle\boldsymbol{u}_{\left(W^{\prime}\right)}\right\rangle(\boldsymbol{x}, t) \cdot \nabla P V_{e}(\boldsymbol{x}, t) \\
& +\left\langle\frac{\partial \boldsymbol{u}_{\left(M, P V_{e}\right)}}{\partial z} \cdot \nabla \theta_{e\left(M, P V_{e}\right)}\right\rangle(\boldsymbol{x}, t)+\left\langle\frac{\partial \boldsymbol{u}_{\left(W^{\prime}\right)}}{\partial z} \cdot \nabla \theta_{e\left(M, P V_{e}\right)}\right\rangle(\boldsymbol{x}, t) \\
& +\left\langle\frac{\partial \boldsymbol{u}_{\left(M, P V_{e}\right)}}{\partial z} \cdot \nabla \theta_{e\left(W^{\prime}\right)}\right\rangle(\boldsymbol{x}, t)+\left\langle\frac{\partial \boldsymbol{u}_{\left(W^{\prime}\right)}}{\partial z} \cdot \nabla \theta_{e\left(W^{\prime}\right)}\right\rangle(\boldsymbol{x}, t) \\
& +\left\langle\xi_{\left(M, P V_{e}\right)}\left(-w_{z\left(W^{\prime}\right)}\right)\right\rangle(\boldsymbol{x}, t)+\left\langle\xi_{\left(W^{\prime}\right)}\left(-w_{z\left(W^{\prime}\right)}\right)\right\rangle(\boldsymbol{x}, t)
\end{aligned}
$$




$$
\begin{aligned}
& +\left\langle w_{x\left(W^{\prime}\right)} v_{z\left(M, P V_{e}\right)}-w_{y_{\left(W^{\prime}\right)}} u_{z\left(M, P V_{e}\right)}\right\rangle(\boldsymbol{x}, t) \\
& +\left\langle w_{x\left(W^{\prime}\right)} v_{z\left(W^{\prime}\right)}-w_{y_{\left(W^{\prime}\right)}} u_{z\left(W^{\prime}\right)}\right\rangle(\boldsymbol{x}, t) .
\end{aligned}
$$

As mentioned above, the details to derive (2.72) and (2.73) are given in Zhang et al. (2021). However, one can see the origin of the coupling terms by direct comparison with the original $\left(M, P V_{e}\right)$ evolution equations given by (2.53) and (2.56). Notice that the terms on the right-hand side of (2.72) and (2.73) are appropriate time averages of terms in (2.53) and (2.56), respectively, after the decomposition of all primary variables $\left(\boldsymbol{u}, \theta_{e}, q_{t}\right)$ into their $\left(M, P V_{e}\right)$ and $(W)$ components. On the other hand, some terms are identically zero following the decomposition and averaging, which can be shown using the explicit structure of the operators in $\mathcal{L}_{*}, \mathcal{L}_{0}$ and $\mathcal{B}$ appearing in (2.64).

\section{Methodology}

Here we further explain the set-up of our numerical simulations and the techniques used to study the different terms in (2.72) and (2.73) at small values of $\epsilon$.

\subsection{Numerical method}

The three-dimensional (3-D) moist Boussinesq equations with two phases of water (vapour and liquid) are simulated in a $2 \pi$-periodic domain using a dealiased, pseudo-spectral code. Calculations with spatial resolutions $128 \times 128 \times 128$ and $256 \times 256 \times 256$ are compared to ensure that the results are insensitive to resolution, e.g. for the $O(1)$ time averages in (2.73) contributing to the evolution of the slow variable $P V_{e}$. The comparison provides confidence in the robustness of our results, especially for the smallest value of the Rossby and Froude numbers $\left(\epsilon \sim 10^{-3}\right)$.

After transferring the physical space equations into Fourier space, a third-order Runge-Kutta time-stepping scheme solves the coupled system of ordinary differential equations (ODEs) resulting from discretization of the wavevector. Linear rotation and buoyancy terms are treated explicitly, and the nonlinear terms are calculated in physical space with implementation of FFTW (http://www.fftw.org/). A pressure solver enforces the incompressibility constraint, and viscous linear terms are included using an integrating factor. A hyperviscosity is used instead of the normal viscosity to induce dissipation only at the smallest scales. For example, in the momentum equation, the hyperviscosity takes the form

$$
(-1)^{p+1} v\left(\nabla^{2}\right)^{p} \boldsymbol{v}
$$

where we use $p=8$. The coefficient $v$ has the structure

$$
v=2.5\left(\frac{E_{v}\left(k_{m}, t\right)}{k_{m}}\right)^{1 / 2} k_{m}^{2-2 p},
$$

where $k_{m}$ is the highest available wavenumber and $E_{v}$ is the kinetic energy in the wavenumber shell associated with $k_{m}$. The spherical shell associated with wavenumber $k_{i}$ includes all wavenumbers satisfying $(i-1) \Delta k<(\boldsymbol{k} \cdot \boldsymbol{k})^{1 / 2} \leq i \Delta k, \Delta k=(2 \pi) / L$, and $L$ is length of the box (in our case $L=2 \pi$ ), where $i=1, \ldots, N$ (in $128^{3}$ spatial resolutions case $N=43$ ).

Similar expressions are used in the equations for equivalent potential temperature $\theta_{e}$ and water $q_{t}$ (Spyksma et al. 2006; Hernandez-Duenas et al. 2013). 


\section{Effects of clouds and phase changes on fast-wave averaging}

\subsection{Discussion of time scales and values of the parameter $\epsilon$}

In the multiscale method to derive the fast-wave-averaging equation (2.64), two time scales (short and long) arise naturally. The model equations have been non-dimensionalized so that $t=O(\epsilon)$ is closely linked to the fast waves (time scale $\tau$ ), while $t=O(1)$ is associated with slow motions (time scale $t$ ). The wave frequencies in the unsaturated and saturated domains are, respectively, given by

$$
\sigma_{u}(\boldsymbol{k})=\frac{\left(F r_{u}^{-2} k_{h}^{2}+R o^{-2} k_{z}^{2}\right)^{1 / 2}}{k}, \quad \sigma_{s}(\boldsymbol{k})=\frac{\left(F r_{s}^{-2} k_{h}^{2}+R o^{-2} k_{z}^{2}\right)^{1 / 2}}{k},
$$

where $F r_{u}, F r_{s}$ are defined in $(2.29 a, b)-(2.30 a, b)$. Given $F r_{s}=F r_{u}=R o=O(\epsilon)$, then $\sigma_{s}=O\left(\epsilon^{-1}\right)$ and the time period of waves in the purely saturated region is $T^{\prime}=2 \pi / \sigma_{s}=$ $O(\epsilon)$. Time steps in the numerical simulations are chosen small enough to simultaneously satisfy the Courant-Friedrichs-Lewy (known as CFL) condition and to resolve the fast-wave oscillations.

We consider the special case $F r_{1}=F r_{2}=R o=\epsilon$ and corresponding $\left(F r_{u}, F r_{s}\right)=$ $(\epsilon / \sqrt{2}, \epsilon),\left(\sigma_{u}, \sigma_{s}\right)=\left(\sqrt{2} \epsilon^{-1}, \epsilon^{-1}\right)$, with $10^{-3} \leq \epsilon \leq 1$. The values of $\left(R o, F r_{u}, F r_{s}\right)$ are monitored in time by calculating the values of these non-dimensional quantities based on their definitions in table 2, where $L=H=2 \pi$ and $U$ is the maximum magnitude of the velocity field. During $O(1)$ time intervals of our decay simulations with hyperviscosity, the values of $\left(R o, F r_{u}, F r_{s}\right)$ do not change significantly, helping to stabilize the value of $\epsilon$ for any given run.

\subsection{Cloud fraction}

During the simulations, the formulae $q_{v}=\min \left(q_{t}, q_{v s}\right), q_{l}=\max \left(0, q_{t}-q_{v s}\right)$ are used to determine vapour $q_{v}$ and liquid water $q_{l}$ from total water $q_{t}$ and saturation threshold $q_{v s}$.

By adjusting the constant parameter $q_{v s}$, one may control the initial cloud fraction quantified by the cloud indicator $H_{s}\left(q_{t}-q_{v s}\right)$. In $\S 4$, the cloud fraction will be calculated as the $L^{1}$ norm of the cloud indicator $H_{s}\left(q_{t}-q_{v s}\right)$. During the $O(1)$-time evolution, for a small amount of initial $\left\|H_{s}\left(q_{t}-q_{v s}\right)\right\|_{1}$ with value less than $\leq 30 \%$ of the domain, the fluctuation of $\left\|H_{s}\left(q_{t}-q_{v s}\right)\right\|_{1}$ over time is quite small $(1 \%-2 \%)$. Thus, for all practical purposes, the cloud fraction can be considered as fixed for the discussion in $\S 4.1$. We note that large initial $\left\|H_{s}\left(q_{t}-q_{v s}\right)\right\|_{1}$, with value $\geq 70 \%$ of the domain, leads to significant fluctuations of $\left\|H_{s}\left(q_{t}-q_{v s}\right)\right\|_{1}$ in time.

In $\S \S 4.1$ and 5.1, the large-scale random initial conditions with $q_{v s}=0.5$ lead to $22 \%$ initial cloud fraction. For the moist bubble set-up discussed in $\$ 4.2$, we use the value $q_{v s}=0.1$ corresponding to cloud fraction $28 \%$. For one of the sensitivity studies, we vary $q_{v s}$ within the context of the large-scale random initial conditions, comparing results for the values $q_{v s}=10,1.5,1,0.5,0,-0.5,-0.8,-1.5,-10$, such that we obtain cloud fractions increasing from $0 \%$ to $100 \%$, respectively.

\subsection{Large-scale, random initial conditions}

For most cases in $\S 4$, we consider decay from large-scale, random initial conditions. The spectral density for all variables $\left(u, v, w, \theta_{e}, q_{t}\right)$ is a Gaussian function given by

$$
F(k)=\epsilon_{f} \frac{\exp \left(-0.5\left(k-k_{f}\right)^{2} / s^{2}\right)}{(2 \pi)^{1 / 2} s}
$$

where $s=1$ is standard deviation, $k_{f}=3$ is the peak wavenumber of the force and $\epsilon_{f}=O(1)$ is the energy input rate. Furthermore, the $k$ values are restricted to the 

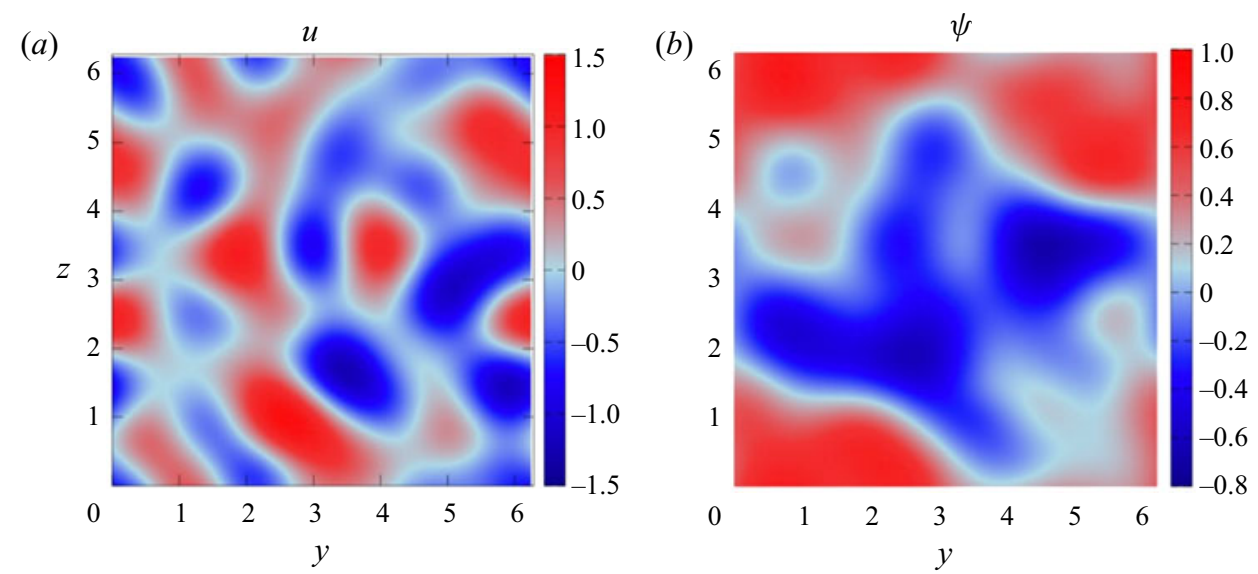

Figure 3. Two-dimensional slices (at $x=\pi$ ) of the zonal velocity $u(a)$ and the stream function $\psi(b)$ at time $t=0$, visualizing the random large-scale initial conditions for these fields.

interval [1, 5]. Upon the change of variable from $\left(u, v, w, \theta_{e}, q_{t}\right)$ to $\left(M, P V_{e}, W_{1}, W_{2}, u_{m}, v_{m}\right)$, the slow $\left(M, P V_{e}\right)$ and fast $(W)$ components have comparable spectral density levels in wavenumbers $[1,5]$.

As a result, the initial conditions contain substantial contributions from waves, and, in this sense, the initial conditions are unbalanced.

For specific choices of the initial distinguished parameter $\epsilon$ (based on maximum magnitude of the initial velocity) and saturation threshold $q_{v s}$, the system evolves according to moist Boussinesq dynamics with phase changes of water. Figure 3 shows 2-D slices of the initial variables $u$ and $\psi$ constructed from aforementioned random initial condition.

\subsection{Evaluation of nonlinear terms in the fast-wave-averaged equation for $P V_{e}$}

As discussed in $\S 2.5$, the $\left(M, P V_{e}\right)$-evolution equations derived from fast-wave averaging have the explicit expressions (2.72) and (2.73). With a closer look at the $P V_{e}$ equation, the nonlinear terms appearing in the right-hand side of (2.73) contain slow-slow, fast-slow, slow-fast, fast-fast interactions.

We would like to analyse each of these nonlinear terms from (2.73), in the limit $\epsilon \rightarrow 0$.

To provide some context for the terms in (2.73), it is helpful to summarize what happens as $\epsilon \rightarrow 0$ in two related cases from earlier literature, and then to compare with the present situation. First, for the case of dry dynamics, only the slow-slow term $\boldsymbol{u}_{(P V)} \cdot \nabla P V$ is non-zero, which means the slowly varying evolution is decoupled from the fast waves (Embid \& Majda 1996, 1998; Majda \& Embid 1998; Majda 2003). Second, for the moist case but with balanced initial conditions (i.e. with initial conditions without waves), only slow-slow terms appear in the $\left(M, P V_{e}\right)$ limiting dynamics (Smith \& Stechmann 2017). However, returning now to the setting of the present paper with initial conditions that are unbalanced, one cannot, a priori, show that all of the fast-fast and fast-slow terms in (2.72) and (2.73) are zero.

Thus, phase changes lead to potential sources of feedback from fast oscillations onto the evolution of the slow modes $\left(M, P V_{e}\right)$. The feedback may originate directly from the fast components $(W)$, or indirectly at phase interfaces through $\left(M, P V_{e}\right)$ inversion, and is manifested through time averages over fast time scales. 


\section{Effects of clouds and phase changes on fast-wave averaging}

Our purpose here is to assess the terms in (2.72) and (2.73) in numerical simulations.

Here we perform a numerical assessment for small values of $\epsilon$, and investigate trends for decreasing $\epsilon$.

For the $P V_{e}$-equation (2.73), the averages to be measured in the simulations at finite $\epsilon$ are the following. Slow-slow (terms 1, 3):

$$
\begin{gathered}
\frac{1}{T} \int_{0}^{T} \boldsymbol{u}_{\left(M, P V_{e}\right)}\left(\boldsymbol{x}, t^{\prime}\right) \cdot \nabla P V_{e}\left(\boldsymbol{x}, t^{\prime}\right) \mathrm{d} t^{\prime}, \\
\frac{1}{T} \int_{0}^{T} \frac{\partial \boldsymbol{u}_{\left(M, P V_{e}\right)}\left(\boldsymbol{x}, t^{\prime}\right)}{\partial z} \cdot \nabla \theta_{e\left(M, P V_{e}\right)}\left(\boldsymbol{x}, t^{\prime}\right) \mathrm{d} t^{\prime} .
\end{gathered}
$$

Fast-slow (terms 2, 4, 9):

$$
\begin{gathered}
\frac{1}{T} \int_{0}^{T} \boldsymbol{u}_{\left(W^{\prime}\right)}\left(\boldsymbol{x}, t^{\prime}\right) \cdot \nabla P V_{e}\left(\boldsymbol{x}, t^{\prime}\right) \mathrm{d} t^{\prime} \\
\frac{1}{T} \int_{0}^{T} \frac{\partial \boldsymbol{u}_{\left(W^{\prime}\right)}\left(\boldsymbol{x}, t^{\prime}\right)}{\partial z} \cdot \nabla \theta_{e\left(M, P V_{e}\right)}\left(\boldsymbol{x}, t^{\prime}\right) \mathrm{d} t^{\prime}, \\
\frac{1}{T} \int_{0}^{T}\left[w_{x\left(W^{\prime}\right)} v_{z\left(M, P V_{e}\right)}-w_{y\left(W^{\prime}\right)} u_{z\left(M, P V_{e}\right)}\right]\left(\boldsymbol{x}, t^{\prime}\right) \mathrm{d} t^{\prime} .
\end{gathered}
$$

Slow-fast (terms 5, 7):

$$
\begin{gathered}
\frac{1}{T} \int_{0}^{T} \frac{\partial \boldsymbol{u}_{\left(M, P V_{e}\right)}\left(\boldsymbol{x}, t^{\prime}\right)}{\partial z} \cdot \nabla \theta_{e\left(W^{\prime}\right)}\left(\boldsymbol{x}, t^{\prime}\right) \mathrm{d} t^{\prime}, \\
\frac{1}{T} \int_{0}^{T}-\xi_{\left(M, P V_{e}\right)} w_{z\left(W^{\prime}\right)}\left(\boldsymbol{x}, t^{\prime}\right) \mathrm{d} t^{\prime} .
\end{gathered}
$$

Fast-fast (terms 6, 8, 10):

$$
\begin{gathered}
\frac{1}{T} \int_{0}^{T}\left\{\frac{\partial \boldsymbol{u}_{\left(W^{\prime}\right)}\left(\boldsymbol{x}, t^{\prime}\right)}{\partial z} \cdot \nabla \theta_{e\left(W^{\prime}\right)}\left(\boldsymbol{x}, t^{\prime}\right)\right. \\
\left.+\left[-\xi_{\left(W^{\prime}\right)} w_{z\left(W^{\prime}\right)}+w_{x\left(W^{\prime}\right)} v_{z\left(W^{\prime}\right)}-w_{y_{\left(W^{\prime}\right)}} u_{z\left(W^{\prime}\right)}\right]\left(\boldsymbol{x}, t^{\prime}\right)\right\} \mathrm{d} t^{\prime} .
\end{gathered}
$$

The numerical quantities we monitor are discrete versions of (3.5)-(3.12) (and similar terms from the $M$-equation) with integration over total time $T$, which means that we average the data in the time window $t \in[0, T]$. Since the time-averaged quantities reach statistically steady state at $T \approx 0.3$, we present the case $T=0.6$ as representative, unless otherwise stated. Note that long-time averages reflect loss of energy due to viscous decay in all variables, obscuring trends. Furthermore, a key diagnostic is the $L^{2}$ norm $\|\langle\cdot\rangle\|_{2}$ of an individual term or group of terms, which will always be normalized by its initial value for comparison between simulations with phase changes and purely saturated simulations without phase changes.

\section{6. $\left(M, P V_{e}\right)$ inversion for finite $\epsilon$}

After each time step of the numerical simulation, the updated state vector $\left(u, v, w, \theta_{e}, q_{t}\right)$ may be used in a post-processing step to find the updated fields $\left(M, P V_{e}, H_{u}, H_{S}\right)$. Then to 
compute the slow components $\boldsymbol{u}_{\left(M, P V_{e}\right)}, \theta_{e}\left(M, P V_{e}\right)$ and $q_{t\left(M, P V_{e}\right)}$, we use the finite- $\epsilon$ version of (2.69) given by

$$
\begin{gathered}
\nabla_{h}^{2} \psi+\frac{\partial}{\partial z}\left[H_{u}\left(\frac{1}{2-\epsilon} \frac{\partial \psi}{\partial z}+\frac{1-\epsilon}{2-\epsilon} M\right)\right] \\
+\frac{\partial}{\partial z}\left[H_{s}\left(\frac{1}{1+\epsilon} \frac{\partial \psi}{\partial z}+\frac{\epsilon}{1+\epsilon} M+(1-2 \epsilon) q_{v s}\right)\right] \\
=P V_{e} .
\end{gathered}
$$

Following inversion of (3.13) to find the streamfuntion $\psi$, the relations $(2.70 a, b)$ are used to obtain $\boldsymbol{u}_{\left(M, P V_{e}\right)}, \theta_{e\left(M, P V_{e}\right)}$. Finally, the definition of $M$ given by (2.52), together with $\theta_{e}\left(M, P V_{e}\right)$, gives $q_{t\left(M, P V_{e}\right)}$.

For the numerical solution of (3.13), a centred-difference method was used, which, owing to the discontinuous coefficients introduced by phase boundaries $\left(H_{u}, H_{s}\right)$, is similar to the ghost fluid approach (Liu, Fedkiw \& Kang 2000; Liu \& Sideris 2003; Tzou \& Stechmann 2019). The conjugate gradient method is then used to solve the discretized symmetric linear system and determine the stream function $\psi$. Here we adopt a simple version of the ghost-fluid method that does not use subcell information about the interface location, together with a Gaussian smoothing of the resulting $\psi$, such that gradient fields may be reliably computed in (3.5)-(3.12). A one-dimensional version of the Gaussian filter is given by

$$
W[\psi](x)=\frac{1}{\sqrt{4 \pi s}} \int_{-\infty}^{\infty} \psi(x-y) \exp \left(\frac{-y^{2}}{4 s}\right) \mathrm{d} y,
$$

where (3.14) is also known as a Weierstrass transformation. For the 3-D version we use the Gaussian-Weierstrass kernel $s=500$ in all three directions for resolution $256^{3}$ ( $s=200$ in all directions for resolution $128^{3}$ ). A simplified test case shows quantitative agreement between this method and a subcell-location version of the ghost-fluid approach.

\section{Results of numerical simulations}

In this section, numerical simulations are used to test the fast-wave averaging theory with phase changes. The most attention will be given to a scenario where the initial conditions are large scale and randomly selected $(\$ 4.1)$. Then some additional sensitivity studies are also conducted to assess the robustness of the results $(\$ 4.2)$.

\subsection{A first assessment: fast-wave averaging with phase changes}

A first assessment will use random initial conditions, which are generated as described above in $\S 3$. The parameter of interest is $\epsilon$, and small values are considered as a numerical investigation of the limit $\epsilon \rightarrow 0$. All other parameters are held fixed, including the averaging window $T=0.6$ and the cloud fraction $\left\|H_{s}\left(q_{t}-q_{v s}\right)\right\|_{1}=22 \%$. Strictly speaking, the cloud indicator $H_{S}$ and distinguished parameter $\epsilon$ are dynamic quantities that evolve with the flow. Nevertheless, the simulation is run for only a time of $O(1)$, and on these time scales, the fluctuations of these two quantities are somewhat small. Hence it is reasonable to use the initial values of $\epsilon$ and cloud fraction $\left\|H_{s}\left(q_{t}-q_{v s}\right)\right\|_{1}$ to represent these two dynamic quantities, and to use these two quantities to help characterize the physical setting of each simulation.

Figure 4 shows the results of the numerical simulations for several different values of $\epsilon$ ranging from 1 to $10^{-3}$. 


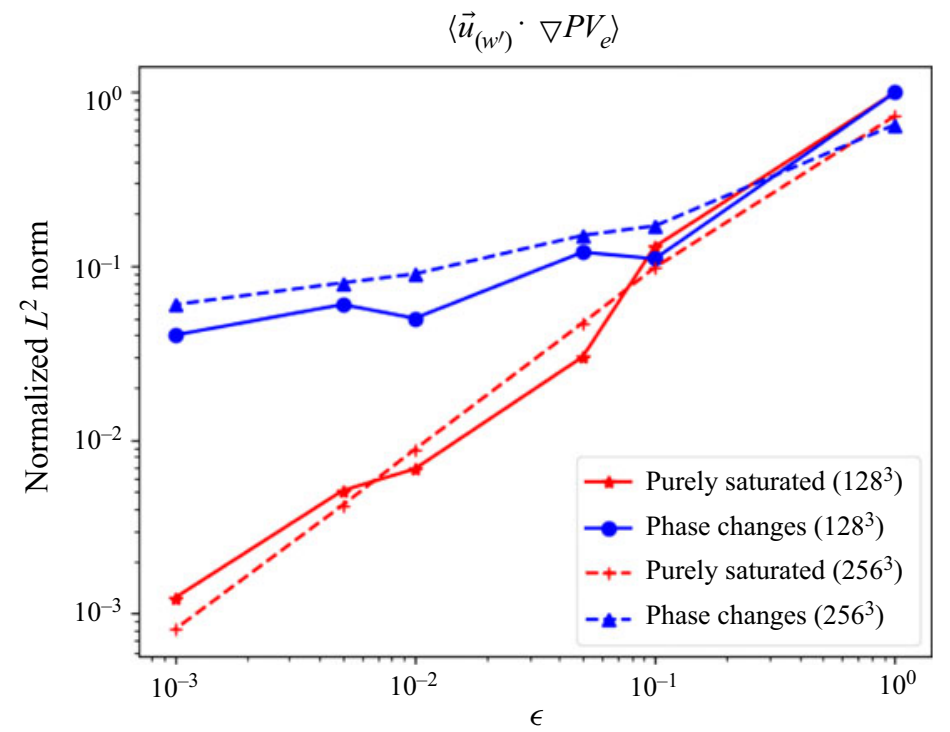

Figure 4. The normalized $L^{2}$ norm of $\left\langle\boldsymbol{u}_{\left(W^{\prime}\right)} \cdot \nabla P V_{e}\right\rangle$ versus the control parameter $\epsilon$ in the range $10^{-3} \leq$ $\epsilon \leq 1$, for decay simulations starting from random large-scale initial conditions. For simulations with phase changes (blue curves), the cloud fraction is $22 \%$. The purely saturated simulations (red curves) show that this particular fast-slow term in the fast-wave-averaged equation for $P V_{e}$ decays roughly linearly with decreasing $\epsilon$. In contrast, these fast-slow terms in the simulations with phase changes decay significantly slower, and will perhaps remain non-zero as $\epsilon \rightarrow 0$.

In this figure, the particular quantity of interest is advection of slow $P V_{e}$ by the fast velocity $\boldsymbol{u}_{\left(W^{\prime}\right)}$. The time average of this advection term is (3.7) and denoted $\left\langle\boldsymbol{u}_{\left(W^{\prime}\right)} \cdot \nabla P V_{e}\right\rangle$, corresponding to one of the fast-slow terms on the right-hand side of the $P V_{e}$ evolution equation (2.73).

The normalized $L^{2}$ norm of this quantity is plotted against the value of $\epsilon$ on a $\log -\log$ plot.

As a baseline for comparison, we also set up a case without phase changes, since the behaviour of the no-phase-change case is already known from past literature (e.g. Embid \& Majda 1996; Majda 2003). For this case, we set up the domain to be purely saturated, so that $H_{s}=1$ and $H_{u}=0$ everywhere. Such a set-up is achieved by appropriately selecting the saturation threshold, $q_{v s}$, as described in $\S 3.3$. For this purely saturated case, the result is shown in figure 4 in red. In this baseline test, the normalized $L^{2}$ norm of $\left\langle\boldsymbol{u}_{\left(W^{\prime}\right)} \cdot \nabla P V_{e}\right\rangle$ decays proportional to $\epsilon$ as $\epsilon \rightarrow 0$. Such a result is in agreement with the dry theory (e.g. Embid \& Majda 1996; Majda 2003), and it provides a demonstration of the soundness of the numerical experiments. Two different numerical resolutions, $128^{3}$ and $256^{3}$, are also shown here to support the numerical robustness. Physically, this plot indicates that the velocity $\boldsymbol{u}_{\left(W^{\prime}\right)}$, which is associated with fast waves, is essentially averaged out for small values of $\epsilon$.

We now turn our attention to the blue curves in figure 4, to assess whether or not the fast waves are also averaged out in the case with phase changes. For $\epsilon=0.1$, the $L^{2}$ norm of $\left\langle\boldsymbol{u}_{\left(W^{\prime}\right)} \cdot \nabla P V_{e}\right\rangle$ is also approximately 0.1 , and its magnitude is similar in the phase-change case and the purely saturated case. Hence, from this $\epsilon=0.1$ experiment, one sees an indication that the fast waves are indeed averaged out, to the extent possible for $\epsilon=0.1$. For smaller values of $\epsilon=10^{-2}$ and $10^{-3}$, the $L^{2}$ norm of $\left\langle\boldsymbol{u}_{\left(W^{\prime}\right)} \cdot \nabla P V_{e}\right\rangle$ remains somewhat small and in the range of roughly $3 \times 10^{-2}$ to $10^{-1}$, and it may continue to 

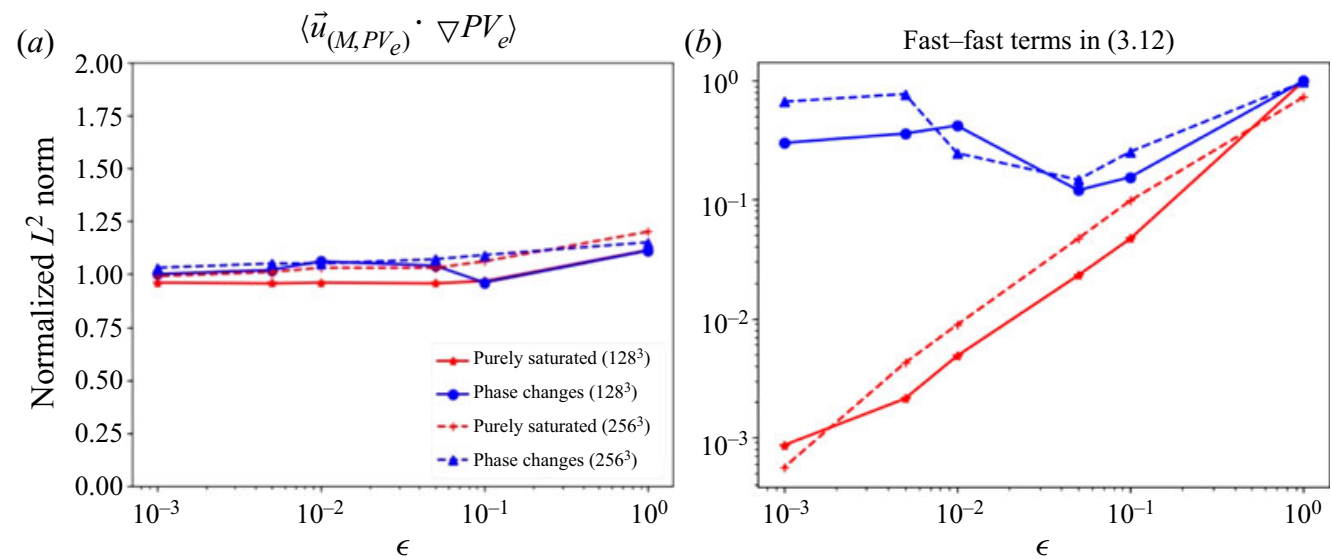

Figure 5. Dependence on $\epsilon$ for the normalized $L^{2}$ norm of terms in the fast-wave-averaged equation for $P V_{e}$. All simulations are decay from random initial conditions. Red curves indicate the purely saturated dynamics, while blue curves are dynamics with phase changes and cloud fraction $22 \%$. (a) In both cases, the slow-slow term $\left\langle\vec{u}_{\left(M, P V_{e}\right)} \cdot \nabla P V_{e}\right\rangle$ remains $O(1)$ as $\epsilon$ is decreased in the range $10^{-3} \leq \epsilon \leq 1$. (b) The sum of the fast-fast terms (3.12) decays in proportion to $\epsilon$ in the purely saturated case (red), but indicates relatively strong feedback onto $P V_{e}$ when phase changes are present (blue).

decay as $\epsilon \rightarrow 0$, but the decay rate is either very slow or tending to zero. In particular, the decay rate is much slower with phase changes (blue) than the decay rate proportional to $\epsilon$ in the purely saturated case (red). The phase changes cause the wave component of the velocity $\boldsymbol{u}_{\left(W^{\prime}\right)}$ to acquire a time-averaged component that is somewhat small, but possibly non-negligible.

For comparison, figure 5 shows the $L^{2}$ norm of other selected terms on the right-hand side of (2.73), contributing to the evolution of $P V_{e}$. In particular, panel $(a)$ examines $\left\langle\boldsymbol{u}_{\left(M, P V_{e}\right)} \cdot \nabla P V_{e}\right\rangle$ for different values of $\epsilon$. This is a slow-slow term, in contrast to the fast-slow term shown earlier in figure 4. For simulations with and without phase changes, and for all values of $\epsilon$, and the (normalized) $L^{2}$ norm of this slow-slow term is approximately equal to unity. Hence, the term $\left\langle\boldsymbol{u}_{\left(M, P V_{e}\right)} \cdot \nabla P V_{e}\right\rangle$ has essentially the same $L^{2}$ norm as its initial value, and is not averaged out. In fact, it is the dominant contribution to $P V_{e}$ advection, in both the phase-change case and the purely saturated case.

Figure $5(b)$ illustrates the dependence on $\epsilon$ for the sum of all fast-fast terms, (3.12), arising in (2.73). The red curves correspond to purely saturated dynamics, and show that the normalized $L^{2}$ norm of the fast-fast terms decays in proportion to $\epsilon$, similar to the fast-slow term in figure 4 . On the other hand, the blue curves indicate relatively strong feedback onto $P V_{e}$ when phase changes are present, again similar to figure 4 . Assessment of figures 4 and 5 together suggest that coupling between fast waves and slow $P V_{e}$ persists in the range $\epsilon \in\left[10^{-3}, 1\right]$, and may not decay to zero as $\epsilon \rightarrow 0$.

On a somewhat subtle issue, we note that figure $5(b)$ displays the sum of all fast-fast nonlinear terms, rather than any single term. In fact, the separate terms do not average to zero, even in the dry or purely saturated dynamics as $\epsilon \rightarrow 0$. This phenomenon can be understood in the single-phase cases by referring to Fourier analysis. More specifically, when there is no phase change present, Fourier analysis shows that the fast-fast nonlinear coefficient $C_{k p q}$ in Fourier space is identically zero (e.g. Smith \& Waleffe 2002). Thus, when viewed in physical space rather than Fourier space, one needs to combine all four terms together to obtain the Fourier inversion of $C_{k p q}$, and to see the budget terms tending 


\section{Effects of clouds and phase changes on fast-wave averaging}
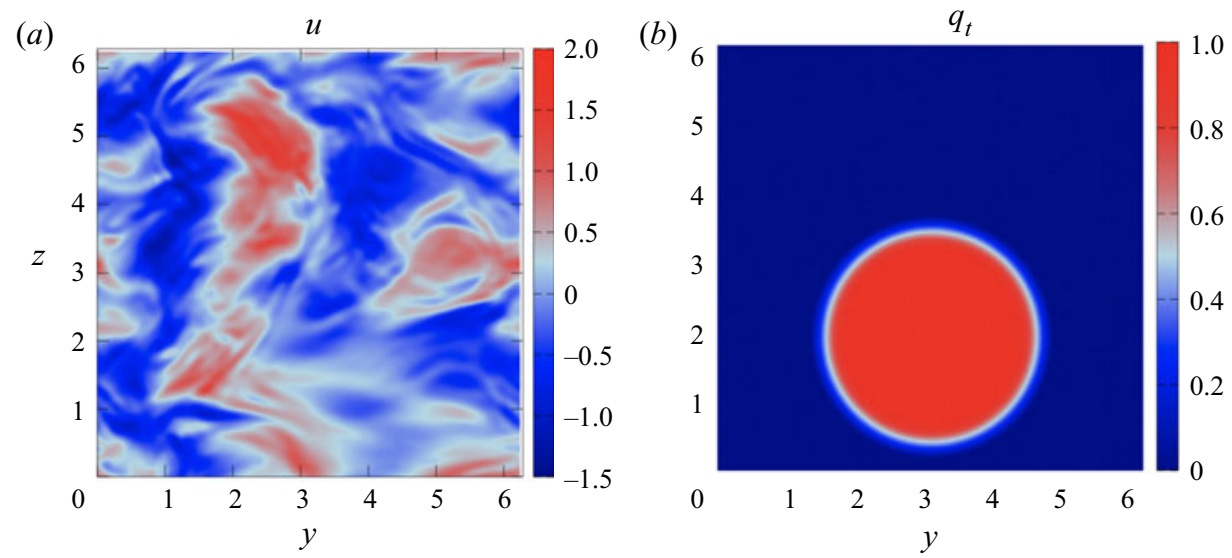

Figure 6. Two-dimensional slices of initial zonal velocity $u(a)$ and total water $q_{t}(b)$ for a sensitivity study. Here the initial conditions involve a turbulent velocity field, along with temperature and water perturbations in the shape of a bubble centred at $x_{0}=(\pi, \pi, 0.625 \pi)$.

toward zero as $\epsilon \rightarrow 0$. This is in contrast to fast-slow terms, such as $\left\|\left\langle\boldsymbol{u}_{\left(W^{\prime}\right)} \cdot \nabla P V_{e}\right\rangle\right\|_{2}$, which is seen in figure 4 to decay proportional to $\epsilon$ as a separate term on its own, for the purely saturated dynamics. In the latter case, it is a different mechanism the Riemann-Lebesgue lemma for time-averaging of fast temporal oscillations - that is responsible for the decay as $\epsilon$ tends toward zero (see, e.g. Majda 2003, § 8.5).

\subsection{Sensitivity studies and robustness tests}

Following the results from the previous $\S 4.1$, naturally one may ask about the robustness of the results. For instance, are the same results seen for different initial conditions? Does the cloud fraction have any impact on the outcome? The above questions are answered in this section using some additional tests.

While the case of randomly selected initial conditions already provides some generality, we now test an initial condition of a different type (see also Spyksma et al. 2006; Wetzel et al. 2020). In particular, the goal is to create some initial conditions that are somewhat simple while also involving the influence of a turbulent flow. Thus, the initial velocity field is generated from large-scale random initial conditions in the absence of buoyancy forces. The forcing and Coriolis parameters are chosen to specify the value of $R o=\epsilon$. The simulation is run to statistically steady-state, thereby providing a dry turbulent state for $\boldsymbol{u}=(u, v, w)$.

At time $t=0$, the initial $\boldsymbol{u}$ is superimposed on stable background temperature and water profiles such that $F r_{1}=F r_{2}=\epsilon$, along with temperature and water perturbations in the shape of a bubble centred at $x_{0}=(\pi, \pi, 0.625 \pi)$. The system is then allowed to evolve according to moist Boussinesq dynamics with phase changes of water. To illustrate the initial conditions, figure 6 shows 2-D slices of the zonal velocity $u$ and the total water $q_{t}$.

After the moist Boussinesq dynamics have run for one non-dimensional time unit to spin-up the new state, including nonlinear interactions between all variables $\boldsymbol{u}, \theta_{e}, q_{t}$, we begin to collect data for a budget analysis of $P V_{e}$ evolution given by (2.73). Specifically, to evaluate terms (3.7) and (3.12), we time-average data from $t \in[1,1.6]$. During this time interval, the cloud fraction is approximately $28 \%$ for the case with phase changes. 

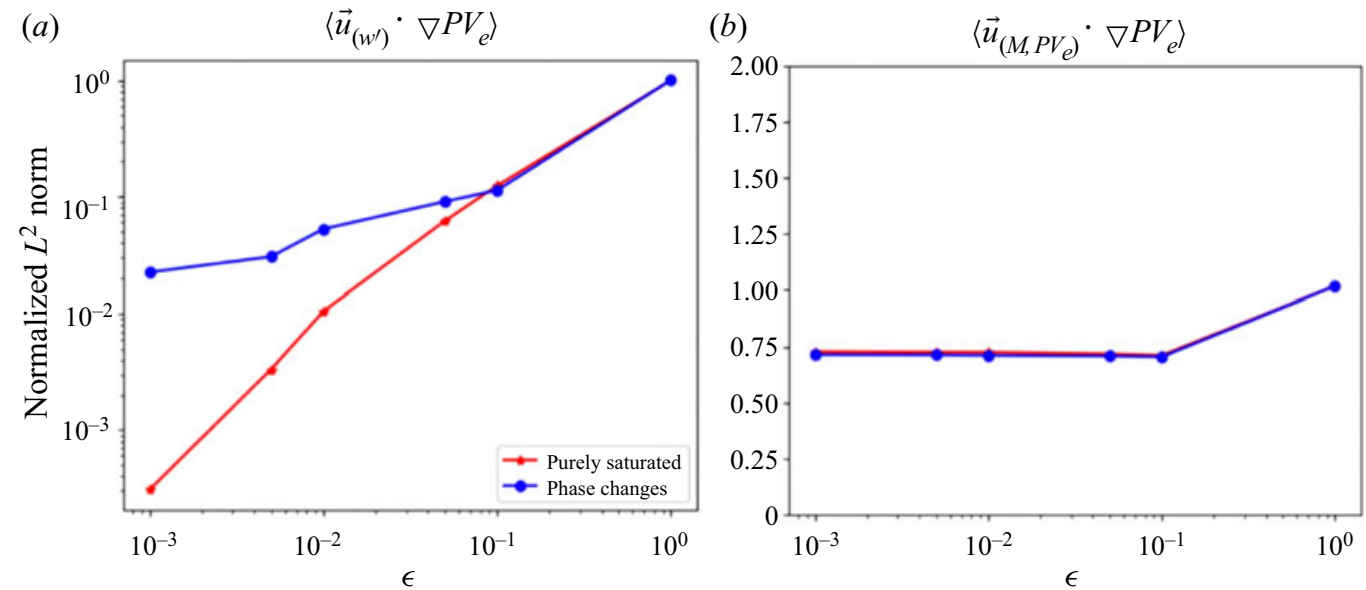

Figure 7. The normalized $L^{2}$ norm of $P V_{e^{-}}$advection terms versus the control parameter $\epsilon$ in the range $10^{-3} \leq$ $\epsilon \leq 1$. Here $(a)\left\langle\boldsymbol{u}_{\left(W^{\prime}\right)} \cdot \nabla P V_{e}\right\rangle$ (a fast-slow term), (b) $\left\langle\boldsymbol{u}_{\left(M, P V_{e}\right)} \cdot \nabla P V_{e}\right\rangle$ (a slow-slow term). Simulations describe decay dynamics starting from initial conditions involving a turbulent velocity field and a water perturbation in the shape of a bubble (resolution $128^{3}$ ). For simulations with phase changes, the cloud fraction is $28 \%$. Differences between the purely saturated (red) and phase-change (blue) curves lead to the same conclusions drawn from figures 4 and 5: for small $\epsilon$ and in a time-averaged sense, the dominant contribution to $P V_{e}$ advection involves the slow velocity $\boldsymbol{u}_{\left(M, P V_{e}\right)}$, and the contribution involving the fast velocity $\boldsymbol{u}_{\left(W^{\prime}\right)}$ is significantly more important when phase changes of water are present.

The simulation is compared with a purely saturated run with $q_{v s}=-10$ and cloud fraction $100 \%$. Results are shown in figure 7 which demonstrate the same conclusions as drawn from the case of random initial conditions, illustrated in earlier figures 4 and 5. Specifically, for small $\epsilon$ and in a time-averaged sense, the dominant contribution to $P V_{e}$ advection involves the slow velocity $\boldsymbol{u}_{\left(M, P V_{e}\right)}$, but the contribution involving the fast velocity $\boldsymbol{u}_{\left(W^{\prime}\right)}$ is significantly more important when phase changes of water are present.

As another set of sensitivity studies, we now discuss the impact of cloud fraction. In particular, recall that figures 4 and 5 showed results for a particular value of cloud fraction of $22 \%$, as well as the purely saturated case where cloud fraction is $100 \%$. We now ask: Do the results change for different values of cloud fraction? For simplicity, attention will be focused on $\left\|\left\langle\boldsymbol{u}_{\left(W^{\prime}\right)} \cdot \nabla P V_{e}\right\rangle\right\|_{2}$ only. For this exploration, we freeze $\epsilon=O\left(10^{-2}\right), T=0.6$ and then vary the initial $q_{v s}$ value to control different initial cloud fractions. The results of the analysis are shown in figure 8 . For the two boundary points of $0 \%$ and $100 \%$ cloud fraction, for which no phase changes are present, notice that the $L^{2}$ norm is $O\left(10^{-2}\right)$ and proportional to $\epsilon$, which indicates that the fast waves are averaged out in these two cases. On the other hand, for other values of cloud fraction between $0 \%$ and $100 \%$, the normalized $\left\|\left\langle\vec{u}_{\left(W^{\prime}\right)} \cdot \nabla P V_{e}\right\rangle\right\|_{2}$ values are larger and do not seem to be proportional to an $\epsilon$ value of $O\left(10^{-2}\right)$, which is consistent with the main conclusion in $\S 4.1$. As a finer detail, notice that the value of $\left\|\left\langle\boldsymbol{u}_{\left(W^{\prime}\right)} \cdot \nabla P V_{e}\right\rangle\right\|_{2}$ increases as cloud fraction increases (away from the two boundary values of $0 \%$ and $100 \%$ cloud fraction). In other words, fast waves appear to be averaged out to a greater degree when the cloud fraction is in the range of $0 \%$ to $20 \%$, which is also the most relevant range for cloud fractions in nature. When the cloud fraction is higher, the fast waves are averaged out less, and the value of $\left\|\left\langle\boldsymbol{u}_{\left(W^{\prime}\right)} \cdot \nabla P V_{e}\right\rangle\right\|_{2}$ is higher. 


\section{Effects of clouds and phase changes on fast-wave averaging}

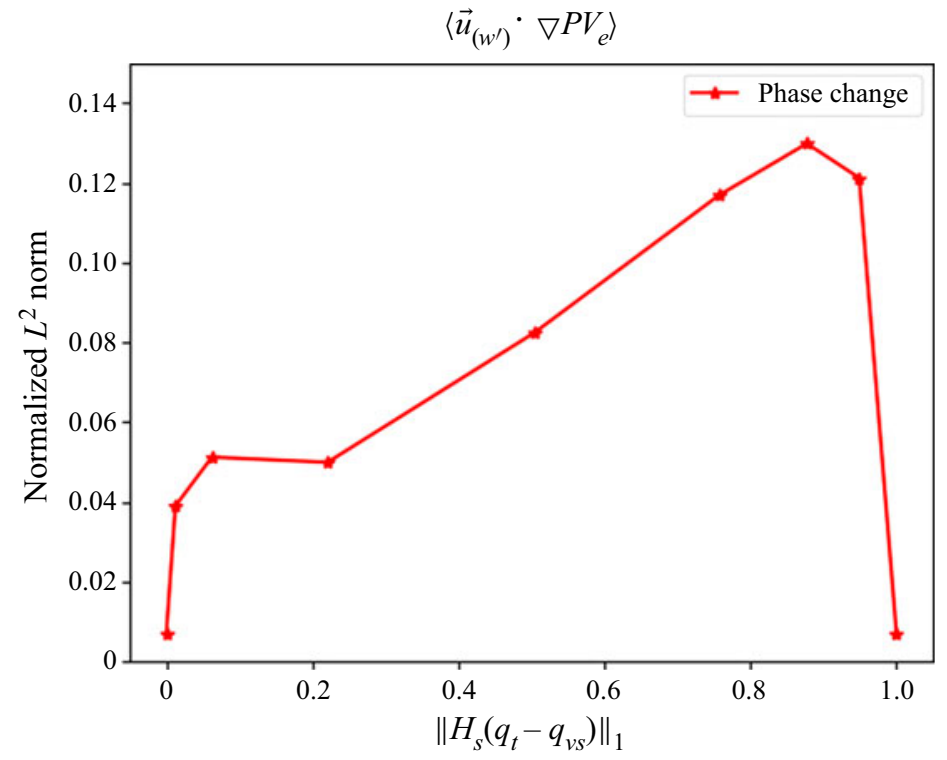

Figure 8. Dependence of the fast-slow term $\left\|\left\langle\boldsymbol{u}_{\left(W^{\prime}\right)} \cdot \nabla P V_{e}\right\rangle\right\|_{2}$ on cloud fraction $\left\|H_{s}\left(q_{t}-q_{v s}\right)\right\|_{1}$. The dynamics evolve from large-scale random initial conditions, the value of $\epsilon=10^{-2}$, and the resolution is $128^{3}$.

\section{Explanation and physical interpretation}

\subsection{A closer look at simulated data}

To explain the mechanism by which phase changes impact fast-wave averaging, we isolate points in the simulations where $O(1)$ time averages are larger than $O(\epsilon)$. We use the simulation with $\epsilon=O\left(10^{-2}\right)$, random large-scale initial conditions and cloud fraction $22 \%$. The goal is to explore the physical properties of these points where the time-average of fast-slow interactions is $O\left(10^{-1}\right)$ instead of proportional to $\epsilon=O\left(10^{-2}\right)$.

Specifically, we monitor the fast-slow term $\left\langle\boldsymbol{u}_{\left(W^{\prime}\right)}\right\rangle(\boldsymbol{x}, t) \cdot \nabla M(\boldsymbol{x}, t)$ in $M$-evolution equation (2.72), starting from random initial conditions. After time averaging, instead of taking the $L^{2}$ norm, we check all points $\left(x_{0}, y_{0}, z_{0}\right)$ in the 3-D domain. A post-processing routine computes the absolute value $\left|(1 / T) \int_{0}^{T} \boldsymbol{u}_{\left(W^{\prime}\right)}\left(\boldsymbol{x}, t^{\prime}\right) \cdot \nabla M\left(\boldsymbol{x}, t^{\prime}\right) \mathrm{d} t^{\prime}\right|$ for $T=0.67$, and highlights every point whose time-averaged absolute value is greater than $O(0.1)$ (see figure $9 c$ ). Meanwhile, in order to observe the relationship between phase changes and such points, we also plot the initial cloud distribution (figure $9 a$ ), and the time-average $\left\langle H_{s}\right\rangle$ of the cloud indicator function $H_{s}$ (figure $9 b$ ). Note that $H_{s}$ is shorthand for $H_{s}\left(q_{t}-q_{v s}\right)$. From the figure, one may observe the following: if the value $\left\langle H_{s}\left(x_{0}, y_{0}, z_{0}\right)\right\rangle$ is near 0 or 1 , then this position $\left(x_{0}, y_{0}, z_{0}\right)$ is away from a phase interface (blue colour in panel $(b)$ ); values $\left\langle H_{s}\left(x_{0}, y_{0}, z_{0}\right)\right\rangle \in[0.2,0.8]$ indicate that this position experiences frequent change of phase (yellow colour in panel $(b)$ ).

A closer look at the hot spots (yellow-red colour) in figure $9(c)$ reveals interesting physical properties of the flow. For a specific point $\left(x_{0}, y_{0}, z_{0}\right)=(64,55,11) *$ $(2 \pi / 128) \approx(3.14,2.70,0.54)$, figure 10 demonstrates how the location alternates between unsaturated and saturated states (figure 10a). During the time $t \in[0,0.67]$, there are 23 time windows with $H_{s}\left(t, x_{0}, y_{0}, z_{0}\right)=1$, and 22 time windows with $H_{s}\left(t, x_{0}, y_{0}, z_{0}\right)=$ 0 . Furthermore, figure $10(b)$ shows the time ratio that the wave spends in those two different states as a function of time window (time ratio $=$ time in each window $/ T$, 

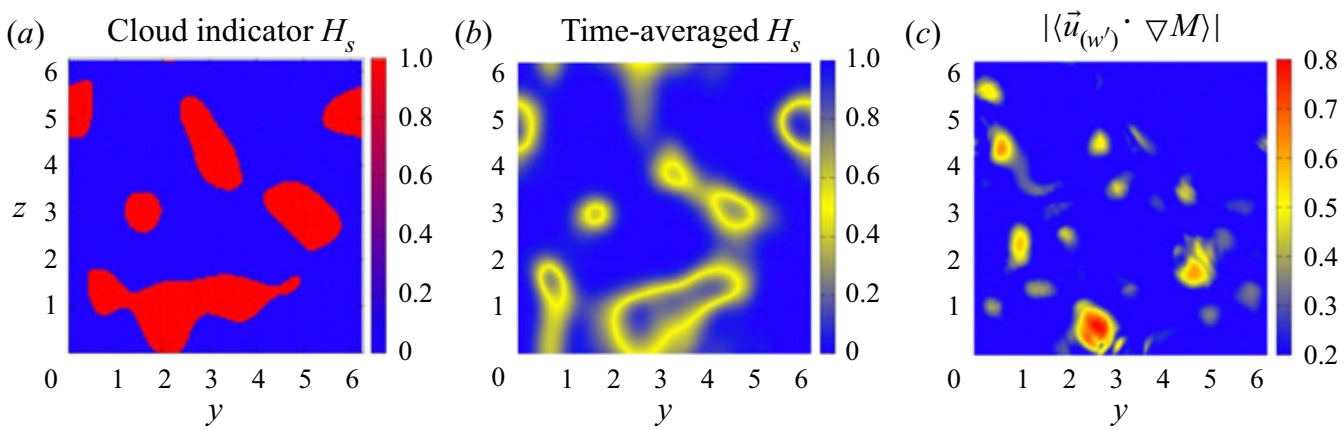

Figure 9. Two-dimensional slices (at $x=\pi$ ) of the initial cloud indicator $H_{s}$ at $t=0(a)$, time-averaged cloud indicator $\left\langle H_{s}\right\rangle(b)$ and absolute value $\left|\left\langle\boldsymbol{u}_{\left(W^{\prime}\right)} \cdot \nabla M\right\rangle\right|$ of a slow-fast term in the fast-time-averaged $M$-equation. Red areas in panel (a) represent clouds (liquid water where $q_{t}>q_{v s}=0.5$ ). Yellow patterns in panel (b) indicate the regions where phase changes happen frequently; blue indicates that the region has remained vapour (value zero) or liquid (value unity) for the duration of the averaging window. Hot spots (yellow to red) in panel (c) indicate high values of the fast-slow coupling term.

where $T=0.67)$. One can see that more time is spent in the saturated state. Quantitatively, $58 \%$ of the time is spent in the saturated state, compared with $42 \%$ of the time in the unsaturated state. Close to a phase boundary, a wave spends more time in the saturated state because the frequencies have the relationship $\left(\sigma_{u}, \sigma_{s}\right)=\left(\sqrt{2} \epsilon^{-1}, \epsilon^{-1}\right)$ (see $(3.3 a, b)$ and recall that $\left.F r_{1}=F r_{2}=\epsilon\right)$. Therefore, the non-zero frequency $\sigma_{u}$ in the unsaturated state is greater than $\sigma_{s}$ in the saturated state. Finally, figure 11 shows that $q_{t}$ has wave fluctuations crossing the saturation threshold, and it spends more time in the saturated state. Other physical variables exhibit similar behaviour.

Since more time is spent in the saturated phase (more time is spent in the wave crest than in the wave valley), $O(1)$ time averages are not zero.

To give a better feel for physical-space variability of the waves, figure 12 shows the wave part $u_{(W)}$ of the horizontal velocity $u=u_{\left(M, P V_{e}\right)}+u_{(W)}$, comparing waves in simulations with phase changes (panels $(a, c, e, g)$, cloud fraction $22 \%$ ) and without phase changes (panels $(b, d, f, h)$, cloud fraction $100 \%)$. The set-up is decay from random initial conditions and the resolution is $128^{3}$. In panels $(a, b)$ corresponding to time $t=0$, notice that the initial conditions of $u_{(W)}$ are slightly different, and this is because the inversion formula for extracting the waves involves Heaviside functions when phase changes are present, but has continuous coefficients when waves are absent. In panels $(c, d)$ and $(e, f)$, one can see that the variation of waves in both scenarios is significant for times $t=O(\epsilon)$. Moreover, smaller scale features are generated during the evolution with phase changes. Finally, panels $(g, h)$ of figure 12 displays the absolute value of $\left\langle u_{(W)}\right\rangle$ for the two cases, using the averaging time $T=0.6$. Corroborating the findings in figure 11, there are locations with significantly higher time-averages $\left\langle u_{(W)}\right\rangle$ when phase boundaries are present (the white and red spots on the left).

\subsection{ODE system}

In this section, we use a model system of ODEs to elucidate the nature of waves that oscillate between unsaturated and saturated regions of the flow. In particular, the ODE model has exact solutions corresponding to waves propagating across phase boundaries, with non-zero time averages, as has been observed in the moist Boussinesq system (figures 9 and 12). 

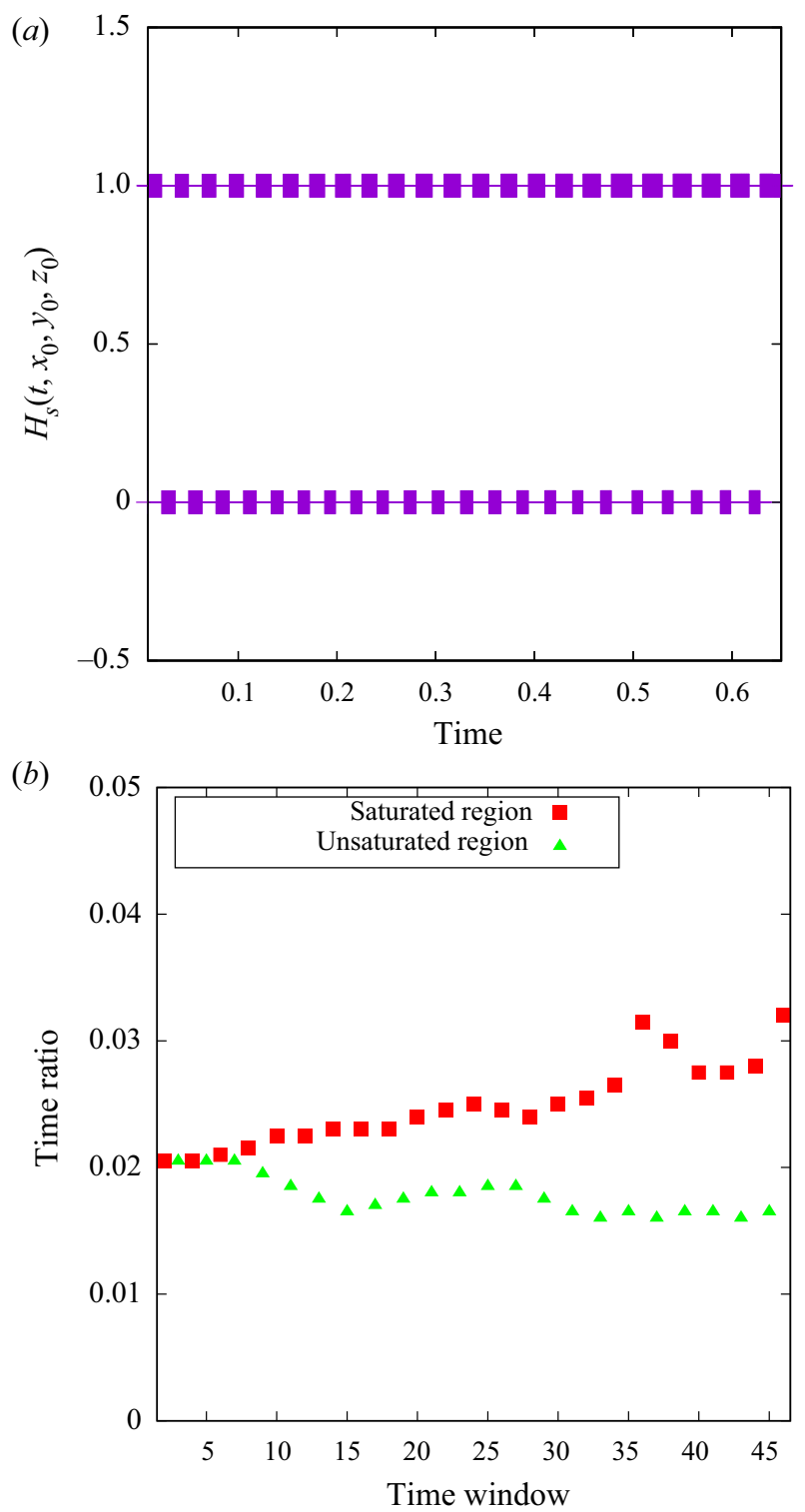

Figure 10. (a) Plot of $H_{S}(t, x)$ versus time $t$ for $t \in[0,0.67]$, with $H_{S}$ measured from a red-spot position $\left(x_{0}, y_{0}, z_{0}\right) \approx(3.14,2.70,0.54)$ in figure 9 . There are 45 total alternating time windows: 23 for the saturated state and 22 for the unsaturated state. Quantitatively, $58 \%$ of the time is spent in the saturated state, compared with $42 \%$ time in the unsaturated state. (b) The green triangles represent the time ratio spent in unsaturated windows, while the red squares correspond to time spent in saturated windows (time ratio $=$ time in each window $/ T$, where $T=0.67$ ).

The ODE model system arises from the 3-D Boussinesq moist dynamics when spatial variations are neglected (see Appendix B),

$$
\frac{\mathrm{d} w}{\mathrm{~d} t}=b=N_{u} b_{u} H_{u}+N_{s} b_{s} H_{s}
$$




\section{Y. Zhang, L.M. Smith and S.N. Stechmann}

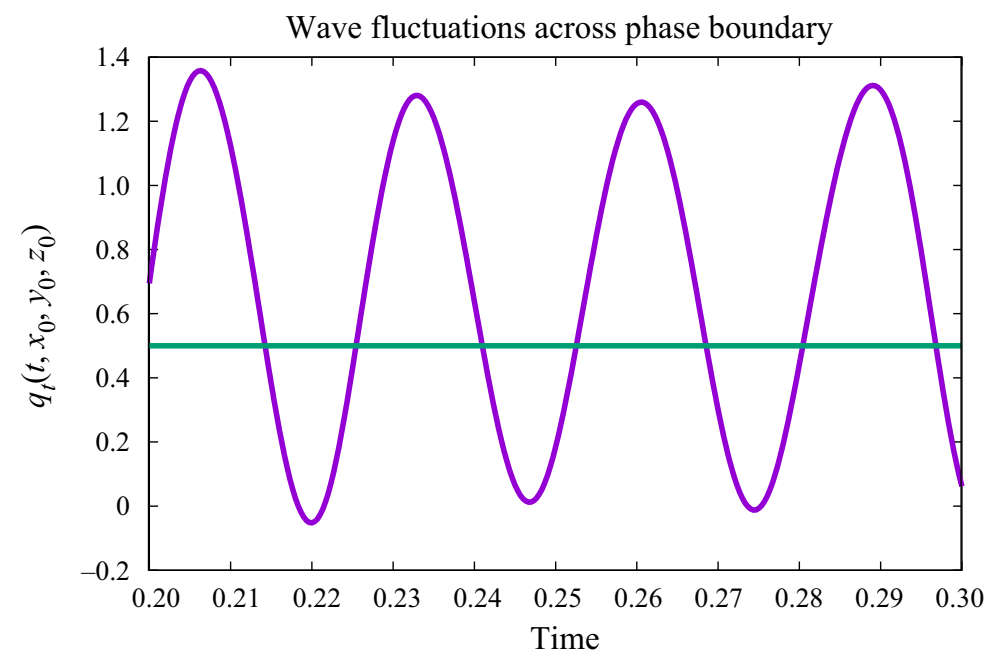

Figure 11. Total water $q_{t}$ versus time $t$ at a fixed red point $\left(x_{0}, y_{0}, z_{0}\right) \approx(3.14,2.70,0.54)$ in figure 9 . The green line is the saturation threshold $q_{v s}=0.5$. The wave (purple curve) has different wavelengths/amplitudes in the saturated and unsaturated regions, located, respectively, above and below the threshold, leading to non-zero $O(1)$ time averages $\left\langle q_{t}\left(t, x_{0}, y_{0}, z_{0}\right)\right\rangle$.

$$
\begin{gathered}
\frac{\mathrm{d} b_{u}}{\mathrm{~d} t}+N_{u} w=0, \\
\frac{\mathrm{d} b_{s}}{\mathrm{~d} t}+N_{s} w=0 .
\end{gathered}
$$

Hence, the ODE system (5.1)-(5.3) describes time variation at a single point in space. Among the velocity components, only the $w$ equation is retained, leading to the simplest set-up that still retains waves. Phase boundaries in (5.1)-(5.3) are identified by the condition $b_{u}=b_{s}$, and the cloud indicator may be written as $H_{s}=H\left(b_{s}-b_{u}\right)$. In analogy with $(3.3 a, b)$ for the Boussinesq system, the waves represented by $(5.1)-(5.3)$ have different frequencies associated with the different phases $\left(b_{u}, b_{s}\right)$ and buoyancy parameters $\left(N_{u}, N_{s}\right)$ (see Durran \& Klemp (1982a) and references therein). This is central for understanding figures 9 and 12.

For the asymptotic theory discussed in the next two sections, we consider the limiting dynamics for buoyancy frequencies $N_{u}, N_{s} \rightarrow \infty$, which is analogous to the limiting dynamics for $\epsilon^{-1} \rightarrow \infty$ in the fast-wave-averaging analysis of the moist Boussinesq system (2.43)-(2.48). We continue to use the special parameter setting $N_{u}^{2}=2 N_{s}^{2}$ as in the numerical simulations, where $N_{u}, N_{s}$ are both positive.

\subsubsection{General solution for $O D E$ system in different phases}

In order to solve (5.1)-(5.3) analytically, the key point is to define the invariant variable $M$.

For the ODE system, the definition of $M$ is $M=N_{u}^{-1} b_{u}-N_{s}^{-1} b_{s}$, which is defined as the linear combination of $b_{u}$ and $b_{s}$ that satisfies* $\mathrm{d} M / \mathrm{d} t=0$. In the earlier PDE system in $\S 2.2, M$ was defined in terms of different variables, $\theta_{e}$ and $q_{t}$, and in non-dimensional units, although the different $M$ definitions can be related via the relationship between the variables $\left(\theta_{e}, q_{t}\right)$ and the variables $\left(b_{u}, b_{s}\right)$ as shown in (2.16) or $(2.51 a, b)$. For both the 
(a)

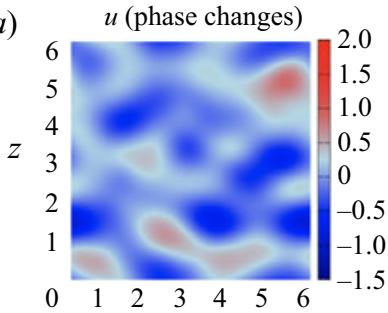

(c)

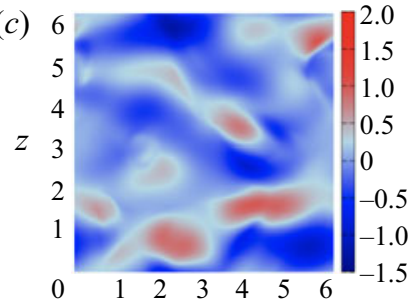

(e)

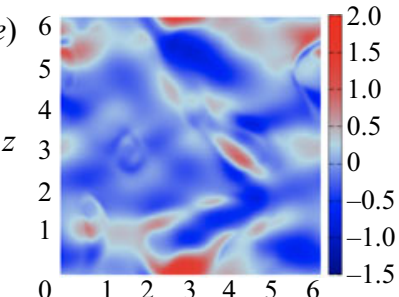

$(g)$

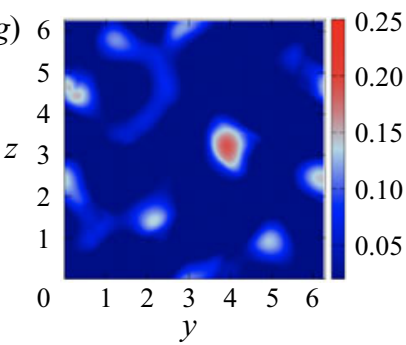

(b)

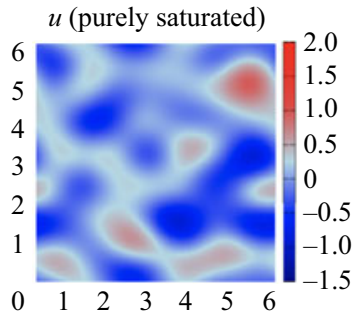

(d)

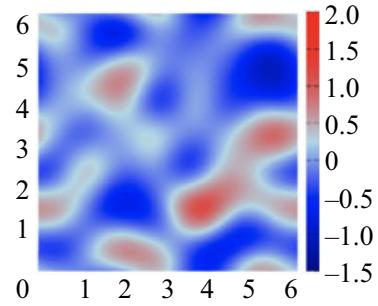

(f)

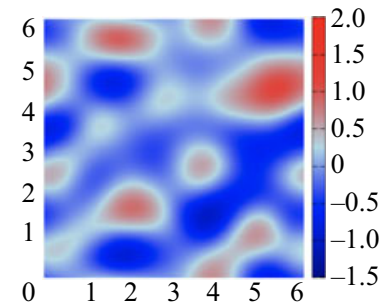

(h)

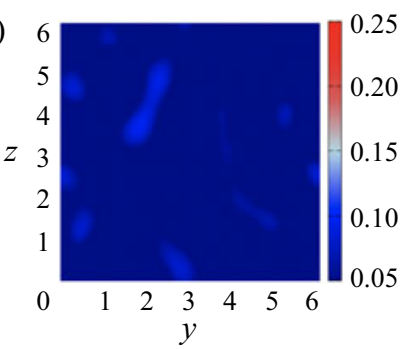

Figure 12. Zonal waves $u_{(W)}$ in decay from random initial conditions during a simulation with phase changes $(a, c, e, g$, cloud fraction $22 \%)$ and a purely saturated simulation $(b, d, f, h$, cloud fraction $100 \%)$. From the first three rows at $t=0,0.02,0.04$, one can see the development of smaller scales when phase changes are present. Panels $(g, h)$ the absolute value of $\left\langle u_{(W)}\right\rangle$, using the averaging $T=0.6$. There are locations with significantly higher time-averages $\left\langle u_{(W)}\right\rangle$ when phase boundaries are present (white and red spots on the left).

ODE system and the PDE system, the motivation for the $M$ definition is the same: find the quantity that satisfies $\mathrm{d} M / \mathrm{d} t=0$ or $\mathrm{D} M / \mathrm{D} t=0$.

Since $\mathrm{d} M / \mathrm{d} t=0$, the value of $M$ is a parameter, and then $b_{s}=\left(N_{s} / N_{u}\right) b_{u}-M N_{s}$. With the replacement of $b_{s}$ in the ODE system, we only need two variables at any given time to describe the evolution as

$$
\begin{gathered}
\frac{\mathrm{d} w}{\mathrm{~d} t}=N_{u} b_{u} H_{u}+N_{s}\left(\frac{N_{s}}{N_{u}} b_{u}-M N_{s}\right) H_{s}, \\
\frac{\mathrm{d} b_{u}}{\mathrm{~d} t}+N_{u} w=0 .
\end{gathered}
$$




\section{Y. Zhang, L.M. Smith and S.N. Stechmann}

The cloud indicator $H_{s}=H\left(b_{s}-b_{u}\right)$ follows from the condition $H_{s}=H\left(q_{t}-q_{v s}\right)$, where $b_{s} \geq b_{u}$ corresponds to a saturated region while $b_{s}<b_{u}$ represents an unsaturated region (Marsico et al. 2019).

For an unsaturated region, $b_{u}>M N_{s} N_{u} /\left(N_{s}-N_{u}\right)$ yields

$$
b_{u}^{\prime \prime}+N_{u}^{2} b_{u}=0
$$

followed by the general solution

$$
b_{u}=c_{u 1} \sin \left(N_{u} t\right)+c_{u 2} \cos \left(N_{u} t\right) .
$$

For a saturated region, $b_{u} \leq M N_{s} N_{u} /\left(N_{s}-N_{u}\right)$ leads to

$$
b_{u}^{\prime \prime}+N_{s}^{2} b_{u}=M N_{s}^{2} N_{u},
$$

followed by the general solution

$$
b_{u}=c_{s 1} \sin \left(N_{s} t\right)+c_{s 2} \cos \left(N_{s} t\right)+M N_{u} .
$$

To find a nonlinear solution that switches phase, as a piecewise sine function, the main idea is to use variables $\left(w, b_{u}\right)$ in the unsaturated phase and then evolve the system forward in time until the saturation condition is reached. After that, we switch to using variables $\left(w, b_{s}\right)$ while in the saturated phase.

\subsubsection{A simple solution example with $M=0$}

A simple solution of the nonlinear wave in the ODE system is now presented, for any $\left(b_{u}\left(t_{0}\right), w\left(t_{0}\right), M\right)$, where $t_{0}$ is the initial time. The interesting case will be an alternating piecewise wave, which crosses the phase boundary repeatedly, passing back and forth between unsaturated and saturated regions. For simplicity, we demonstrate using an example with $M=0$, which means that the phase boundary is exactly at $b_{u}=0$.

Without loss of generality, assume an initial buoyancy exactly at the phase boundary such that $b_{u}\left(t_{0}\right)=0$, and set the initial vertical velocity $w\left(t_{0}\right)=a$, where $a$ is an arbitrary positive number. Note that $b_{u}^{\prime}\left(t_{0}\right)=-N_{u} w\left(t_{0}\right)=-N_{u} a<0$, which indicates that the solution will enter the saturated region first. The analytical solution for this initial condition is given by

$$
b_{u}(t)= \begin{cases}-\frac{a N_{u}}{N_{s}} \sin \left(N_{s}\left(t-t_{0}\right)\right) & t \in\left[t_{0}+n \mathcal{T}, t_{0}+\frac{\pi}{N_{s}}+n \mathcal{T}\right], \\ a \sin \left(N_{u}\left(t-t_{0}-\frac{\pi}{N_{s}}\right)\right) & t \in\left[t_{0}+\frac{\pi}{N_{s}}+n \mathcal{T}, t_{0}+(n+1) \mathcal{T}\right],\end{cases}
$$

where $\mathcal{T}=\pi / N_{u}+\pi / N_{s}$ and $n=0,1,2, \ldots$ Then, after one half-period at $t=t_{0}+$ $\pi / N_{s}$, the solution meets the phase interface at $b_{u}=0$ with $b_{u}\left(t_{0}+\pi / N_{s}\right)=0, b_{u}^{\prime}\left(t_{0}+\right.$ $\left.\pi / N_{s}\right)=a N_{u}>0$, which is the starting point for wave propagation in the unsaturated region.

Using (5.10), we can now calculate the time-averaged value $\left|(1 / T) \int_{0}^{T} b_{u}(t) \mathrm{d} t\right|$, to determine if there is a non-zero average when a phase boundary is present. Without loss of generality, we choose $a=1$ and $N_{u}=\sqrt{2} N_{s}$. On the one hand, consider the purely unsaturated case without phase changes, in which case the analytical solution for $b_{u}(t)$ is a simple sine function with frequency $N_{u}$. In that case, for fixed averaging interval $T$, 


\section{Effects of clouds and phase changes on fast-wave averaging}

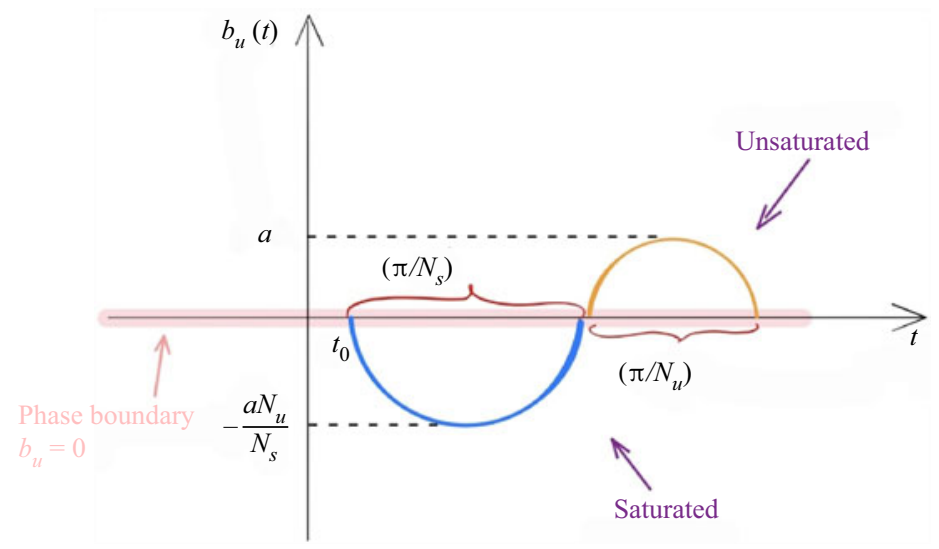

Figure 13. Sketch of the piecewise solution to (5.10) for $M=0$ such that the phase interface is at $b_{u}=0$.

the average $\left|(1 / T) \int_{0}^{T} b_{u}(t) \mathrm{d} t\right| \leq 2 / N_{u} T \rightarrow 0$ as $N_{u} \rightarrow \infty$. On the other hand, if the phase interface is present, then we find the following relation:

$$
\frac{2}{\pi(1+\sqrt{2})}-\frac{2}{N_{u} T} \leq\left|\frac{1}{T} \int_{0}^{T} b_{u}(t) \mathrm{d} t\right| \leq \frac{2}{\pi(1+\sqrt{2})}+\frac{2}{N_{u} T}, \quad N_{u} \rightarrow \infty,
$$

which is strictly bounded away from zero. Figure 13 displays the solution (5.10), and clearly illustrates how different frequencies $N_{u} \neq N_{s}$ lead to non-zero time average of $b_{u}(t)$ as in (5.11). Most importantly, the same essential mechanism is observed in the 3-D Boussinesq simulations, as seen in figure 11.

\section{Concluding discussion}

Phase changes of water in atmospheric flows are as fundamental as the presence of fast inertia-gravity waves generated by the effects of rotation and stable stratification. Here, all these effects are combined in an idealized model with a Boussinesq dynamical core and simplified thermodynamics allowing for phase changes of water, from vapour to liquid and vice versa. We conduct moist Boussinesq simulations designed to support asymptotic theory in the limit of vanishing Rossby and Froude numbers: $\operatorname{Ro}=F_{1}=\mathrm{Fr}_{2}=\epsilon \rightarrow 0$ (asymptotically large rotation and strong stable stratification). The theory separates the state space of the Boussinesq dynamics into fast waves evolving on short times $t=O(\epsilon)$, and slowly varying $\left(M, P V_{e}\right)$ components evolving on times $t=O(1)$. Furthermore, the initial conditions are assumed to contain fast waves. Then the central goal is to assess the coupling between fast and slow components on $O(1)$ time scales as $\epsilon \rightarrow 0$. Such coupling is explicitly represented by terms in the fast-wave-averaging equation (2.64). For example, in the $P V_{e}$ equation, (2.73), the fast-slow, slow-fast and fast-fast terms, (3.7)-(3.12), do not a priori vanish in an environment with phase changes. This situation is in contrast to the case of dry dynamics, for which rigorous proofs establish decoupling between the dry $P V$ and the fast waves (Embid \& Majda 1996, 1998; Majda \& Embid 1998; Majda 2003). On the other hand, all of the terms (3.5)-(3.12) can be measured in numerical simulations at finite $\epsilon$, and thus our companion numerical calculations investigate trends in their behaviours for decreasing $\epsilon$ in the range $10^{-3} \leq \epsilon \leq 1$.

The main suite of simulations starts from random, large-scale initial conditions and varies the distinguished parameter $\epsilon$, along with the saturation threshold $q_{v s}$ (which 


\section{Y. Zhang, L.M. Smith and S.N. Stechmann}

determines the resulting cloud fraction). For example, when the cloud fraction is roughly $22 \%$ of the domain, the simulations show that the sum of the fast-fast terms, (3.12), does not decay with $\epsilon$ (figure 5), and the fast-slow term $\left\|\left\langle\boldsymbol{u}_{\left(W^{\prime}\right)} \cdot \nabla P V_{e}\right\rangle\right\|_{2}$ decays significantly slower with $\epsilon$ than the analogous term in the dry dynamics (figure 4). For fixed $\epsilon$, robustness studies indicate that fast-slow coupling increases with cloud fraction, up to cloud fractions of at least $80 \%$ of the domain (figure 8). Altogether, the results suggest that coupling of $\left(M, P V_{e}\right)$ dynamics with fast-waves may persist as $\epsilon \rightarrow 0$ when the cloud fraction is in the range [10\%,85\%].

The limiting equations (2.72) and (2.73) for $\left(M, P V_{e}\right)$ may contain non-zero averages arising mathematically from two sources. Feedback from waves onto $\left(M, P V_{e}\right)$ may originate directly from the fast components $(W)$, or indirectly at phase interfaces through $\left(M, P V_{e}\right)$ inversion, (2.69). To gain insight into the behaviour of waves near a phase interface, we use a coupled ODE system for vertical velocity $w(t)$, together with unsaturated buoyancy $b_{u}(t)$ and saturated buoyancy $b_{s}(t)$. In this model, the saturation condition is given by $b_{u}=b_{s}$, and the saturation threshold is given in terms of the parameter $M$. The buoyancies $b_{u}$ and $b_{s}$ evolve according to different oscillator equations associated with different frequencies $N_{u}$ and $N_{s}$. To solve the ODE system from an unsaturated initial $b_{u}$, one may first integrate the oscillator equation for $b_{u}$ until the saturation condition $b_{u}=b_{s}$ is reached, and then proceed to integrate the oscillator equation for $b_{s}$ until $b_{s}=b_{u}$, and so on. For $N_{u} \neq N_{s}$, the exact solution consists of a piecewise wave solution as in figure 13. By virtue of the different frequencies and wavelengths on either side of the phase boundary, the time average of $b_{u}$ over $t=O(1)$ is bounded away from zero for asymptotically large $N_{u}$. Such piecewise waves are indeed observed in the Boussinesq simulations, e.g. as seen in the solution for $q_{t}\left(t, \boldsymbol{x}_{0}\right)$ for fixed location $\boldsymbol{x}_{0}$ close to a phase boundary (figure 11).

For Boussinesq dynamics with phase changes, the formal asymptotic theory, PDE numerical simulations and model-ODE exact solutions together indicate that feedback from waves onto slowly varying flow components may have an impact even as the Rossby and Froude numbers tend to zero. Especially given the importance of phase changes for atmospheric applications, several follow-up studies are planned. Theoretically, the longer-term goal is to perform rigorous analysis in which the phase boundaries are determined as part of the solution. Future numerical simulations will investigate convergence of the fast-wave-averaging equations to the PQG equations, starting from balanced initial conditions, as well as the effects of fast-wave coupling for turbulence steady states with phase changes.

In terms of the physics of clouds, the set-up here was basic and only considered the vapour-liquid phase change. It would be interesting in the future to investigate the influence of other aspects of cloud physics, such as rain water $q_{r}$, cloud ice $q_{i}$, or number density $n_{c}$ of cloud droplets (e.g. Kessler 1969; Lin, Farley \& Orville 1983; Seifert \& Beheng 2001, 2006). To do so, it has been suggested by Smith \& Stechmann $(2017, \S 9)$ that additional slow $M$ variables arise in association with additional cloud variables such as $q_{r}$, $q_{i}$ or $n_{c}$ (see also Wetzel et al. 2019b,2020). Even in the basic set-up of the present paper, the fast waves already have an influence on the slow components of the flow. If additional cloud physics is considered, one might expect to see additional coupling between fast waves and slow components.

Funding. Funding is provided by the National Science Foundation NSF-DMS-1907667.

Declaration of Interests. The authors report no conflict of interest. 


\section{Effects of clouds and phase changes on fast-wave averaging}

\section{Author ORCIDs.}

다 Yeyu Zhang https://orcid.org/0000-0002-7954-8348.

\section{Appendix A. The fast-wave-averaged equation with phase changes}

Here we provide a sketch of the steps to derive the fast-wave-averaged equation (2.64), starting from the moist Boussinesq system (2.61)-(2.62), including the effects of phase changes represented by the Heaviside functions $H_{u}(\boldsymbol{x}, t), H_{s}(\boldsymbol{x}, t)$. For the purposes of fast-wave averaging, one may consider a known evolution from initial conditions containing waves. Then the goal is to determine the dynamical coupling between the slowly varying and fast-wave components embedded within the evolution (2.61)-(2.62). The Heaviside functions are part of the known solution, and are therefore treated as given functions of $(\boldsymbol{x}, t)$.

The idea is to take advantage of the small parameter $\epsilon$, and thus to use a two-time scale asymptotic expansion in powers of $\epsilon$, where the fast time scale is $\tau=t / \epsilon$. Since the phase boundaries $H_{u}, H_{s}$ are determined by the complete (thermo)dynamics, they have both slowly varying and fast components. A main new element of the formulation is the $\tau$ dependence in the linear operator $\mathcal{L}_{*}(t, \tau)$ in $(2.62)$, leading to differences from the analysis for the dry Boussinesq equations.

The two-time-scale expansion of the state vector

$$
\boldsymbol{v}^{\epsilon}(\boldsymbol{x}, t, \tau)=\left.\boldsymbol{v}^{0}(\boldsymbol{x}, t, \tau)\right|_{\tau=t / \epsilon}+\left.\epsilon \boldsymbol{v}^{1}(\boldsymbol{x}, t, \tau)\right|_{\tau=t / \epsilon}+\cdots
$$

is inserted into the system

$$
\frac{\partial \boldsymbol{v}}{\partial t}+\epsilon^{-1} \mathcal{L}_{*}(t, \tau)(\boldsymbol{v})+\mathcal{L}_{0}(t, \tau)(\boldsymbol{v})+\mathcal{B}(\boldsymbol{v}, \boldsymbol{v})=0 .
$$

Collecting $O\left(\epsilon^{-1}\right)$ terms leads to the balance

$$
\frac{\partial \boldsymbol{v}^{0}}{\partial \tau}+\mathcal{L}_{*}(t, \tau)\left(\boldsymbol{v}^{0}\right)=0
$$

with solutions

$$
\boldsymbol{v}^{0}(\boldsymbol{x}, t, \tau)=\exp \left(-\int_{0}^{\tau} \mathcal{L}_{*}\left(t, \tau^{\prime}\right) \mathrm{d} \tau^{\prime}\right) \bar{v}(\boldsymbol{x}, t),
$$

where $\bar{v}(\boldsymbol{x}, t)$ is the initial condition for (A3) and thus depends on $(\boldsymbol{x}, t)$, but not on $\tau$. The next-order $O\left(\epsilon^{0}\right)$ balance yields

$$
\frac{\partial \boldsymbol{v}^{1}}{\partial \tau}+\mathcal{L}_{*}(t, \tau)\left(\boldsymbol{v}^{1}\right)=-\left(\frac{\partial \boldsymbol{v}^{0}}{\partial t}+\mathcal{L}_{0}(t, \tau)\left(\boldsymbol{v}^{0}\right)+\mathcal{B}\left(\boldsymbol{v}^{0}, \boldsymbol{v}^{0}\right)\right),
$$

and one may integrate with respect to $\tau$, keeping $t$ fixed as $\epsilon \rightarrow 0$. The calculus is straightforward, though slightly more complicated than for the dry case, and for illustration we provide details for the $\partial \boldsymbol{v}^{0} / \partial t$ term on the right-hand side of (A5). The standard integrating factor method gives

$$
\begin{gathered}
\boldsymbol{v}^{1}=-\exp \left(-\int_{0}^{\tau} \mathcal{L}_{*}\left(t, \tau^{\prime}\right) \mathrm{d} \tau^{\prime}\right) \int_{0}^{\tau} \exp \left(\int_{0}^{s} \mathcal{L}_{*}\left(t, s^{\prime}\right) \mathrm{d} s^{\prime}\right) \\
\times \frac{\partial\left(\exp \left(-\int_{0}^{s} \mathcal{L}_{*}\left(t, s^{\prime}\right) \mathrm{d} s^{\prime}\right) \bar{v}\right)}{\partial t} \mathrm{~d} s+\cdots
\end{gathered}
$$




$$
\begin{gathered}
=-\exp \left(-\int_{0}^{\tau} \mathcal{L}_{*}\left(t, \tau^{\prime}\right) \mathrm{d} \tau^{\prime}\right) \int_{0}^{\tau} \exp \left(\int_{0}^{s} \mathcal{L}_{*}\left(t, s^{\prime}\right) \mathrm{d} s^{\prime}\right)\left[\frac{\partial\left(\exp \left(-\int_{0}^{s} \mathcal{L}_{*}\left(t, s^{\prime}\right) \mathrm{d} s^{\prime}\right)\right)}{\partial t} \bar{v}\right. \\
\left.\quad+\frac{\partial \bar{v}}{\partial t} \exp \left(-\int_{0}^{s} \mathcal{L}_{*}\left(t, s^{\prime}\right) \mathrm{d} s^{\prime}\right)\right] \mathrm{d} s+\cdots \\
=-\exp \left(-\int_{0}^{\tau} \mathcal{L}_{*}\left(t, \tau^{\prime}\right) \mathrm{d} \tau^{\prime}\right) \tau \frac{\partial \bar{v}}{\partial t}-\exp \left(-\int_{0}^{\tau} \mathcal{L}_{*}\left(t, \tau^{\prime}\right) \mathrm{d} \tau^{\prime}\right) \\
\times \int_{0}^{\tau}\left(-\int_{0}^{s} \frac{\partial \mathcal{L}_{*}\left(t, s^{\prime}\right)}{\partial t} \mathrm{~d} s^{\prime}\right) \bar{v} \mathrm{~d} s+\cdots
\end{gathered}
$$

where $\boldsymbol{v}^{1}=\boldsymbol{v}^{1}(\boldsymbol{x}, t, \tau)$ and $\bar{v}=\bar{v}(\boldsymbol{x}, t)$. To arrive at the final form (A6), we have used the explicit structure of the operators

$$
\left(-\int_{0}^{s} \frac{\partial \mathcal{L}_{*}\left(t, s^{\prime}\right)}{\partial t} \mathrm{~d} s^{\prime}\right) \text { and } \exp \left(-\int_{0}^{s} \frac{\partial \mathcal{L}_{*}\left(t, s^{\prime}\right)}{\partial t} \mathrm{~d} s^{\prime}\right) .
$$

Integration of (A5) including all the terms leads to the full equation for $\bar{v}^{1}$, given by

$$
\begin{gathered}
\boldsymbol{v}^{1}=\left.\exp \left(-\int_{0}^{\tau} \mathcal{L}_{*}\left(t, \tau^{\prime}\right) \mathrm{d} \tau^{\prime}\right) \boldsymbol{v}^{1}\right|_{\tau=0}-\exp \left(-\int_{0}^{\tau} \mathcal{L}_{*}\left(t, \tau^{\prime}\right) \mathrm{d} \tau^{\prime}\right) \\
\times\left\{\tau \frac{\partial \bar{v}}{\partial t}-\int_{0}^{\tau}\left(\int_{0}^{s} \frac{\partial \mathcal{L}_{*}\left(t, s^{\prime}\right)}{\partial t} \mathrm{~d} s^{\prime}\right) \bar{v} \mathrm{~d} s\right. \\
+\int_{0}^{\tau} \exp \left(\int_{0}^{s} \mathcal{L}_{*}\left(t, s^{\prime}\right) \mathrm{d} s^{\prime}\right)\left[\mathcal{L}_{0}(t, s)\left(\exp \left(-\int_{0}^{s} \mathcal{L}_{*}\left(t, s^{\prime}\right) \mathrm{d} s^{\prime}\right) \bar{v}\right)\right. \\
\left.\left.+\mathscr{B}\left(\exp \left(-\int_{0}^{s} \mathcal{L}_{*}\left(t, s^{\prime}\right) \mathrm{d} s^{\prime}\right) \bar{v}, \exp \left(-\int_{0}^{s} \mathcal{L}_{*}\left(t, s^{\prime}\right) \mathrm{d} s^{\prime}\right) \bar{v}\right)\right] \mathrm{d} s\right\} .
\end{gathered}
$$

Next, to preserve the ordering of the expansion (A1), one must impose the condition $\boldsymbol{v}^{1}=o(\tau)$, thereby constraining $\partial \bar{v} / \partial t$ appearing on the right-hand side of (A8). This sublinear-in-time growth condition is also called the fast-wave-averaged equation. In the limit $\epsilon \rightarrow 0, \tau=t / \epsilon \rightarrow \infty$ with $t=O(1)$, the fast-wave-averaging equation is thus given by

$$
\begin{aligned}
& \frac{\partial \bar{v}(\boldsymbol{x}, t)}{\partial t}=\lim _{\tau \rightarrow \infty} \frac{1}{\tau} \int_{0}^{\tau}\left\{\left(\int_{0}^{s} \frac{\partial \mathcal{L}_{*}\left(t, s^{\prime}\right)}{\partial t} \mathrm{~d} s^{\prime}\right) \bar{v}-\exp \left(\int_{0}^{s} \mathcal{L}_{*}\left(t, s^{\prime}\right) \mathrm{d} s^{\prime}\right)\right. \\
& \times\left[\mathcal{L}_{0}(t, s)\left(\exp \left(-\int_{0}^{s} \mathcal{L}_{*}\left(t, s^{\prime}\right) \mathrm{d} s^{\prime}\right) \bar{v}\right)\right. \\
& \left.\left.+\mathcal{B}\left(\exp \left(-\int_{0}^{s} \mathcal{L}_{*}\left(t, s^{\prime}\right) \mathrm{d} s^{\prime}\right) \bar{v}, \exp \left(-\int_{0}^{s} \mathcal{L}_{*}\left(t, s^{\prime}\right) \mathrm{d} s^{\prime}\right) \bar{v}\right)\right]\right\} \mathrm{d} s,
\end{aligned}
$$

where the operators $\mathcal{L}_{*}, \mathcal{L}_{0}$ and $\mathcal{B}$ are defined in $\S 2$ of (Zhang et al. 2021).

\section{Appendix B.}

In this appendix, we expand on the derivation of the ODE system discussed in $\S 5.2$. We use the dimensional version of (2.16) and write the dimensional moist Boussinesq system 
Effects of clouds and phase changes on fast-wave averaging

using variables $b_{u}, b_{s}$ instead of $\theta_{e}, q_{t}$ (Smith \& Stechmann 2017; Marsico et al. 2019),

$$
\begin{gathered}
\frac{\mathrm{D}_{h} \boldsymbol{u}_{h}}{\mathrm{D} t}+w \frac{\partial \boldsymbol{u}_{h}}{\partial z}+f \boldsymbol{u}_{h}^{\perp}+\nabla_{h} \phi=0, \\
\frac{\mathrm{D}_{h} w}{\mathrm{D} t}+w \frac{\partial w}{\partial z}+\frac{\partial \phi}{\partial z}=\left(N_{u} b_{u} H_{u}+N_{s} b_{s} H_{s}\right), \\
\nabla_{h} \cdot \boldsymbol{u}_{h}+\frac{\partial w}{\partial z}=0, \\
\frac{\mathrm{D}_{h} b_{u}}{\mathrm{D} t}+w \frac{\partial b_{u}}{\partial z}+N_{u} w=0, \\
\frac{\mathrm{D}_{h} b_{s}}{\mathrm{D} t}+w \frac{\partial b_{s}}{\partial z}+N_{s} w=0 .
\end{gathered}
$$

Neglecting spatial variations leads to the ODE system

$$
\begin{gathered}
\frac{\mathrm{d} \boldsymbol{u}_{h}}{\mathrm{~d} t}+f \boldsymbol{u}_{h}^{\perp}=0, \\
\frac{\mathrm{d} w}{\mathrm{~d} t}=N_{u} b_{u} H_{u}+N_{s} b_{s} H_{s}, \\
\frac{\mathrm{d} b_{u}}{\mathrm{~d} t}+N_{u} w=0, \\
\frac{\mathrm{d} b_{s}}{\mathrm{~d} t}+N_{s} w=0 .
\end{gathered}
$$

Hence, the ODE system (B6)-(B9) describes time variation at a single point in space. Finally, among the velocity components $(u, v, w)$, we only keep the $w$ equation, which is the simplest set-up that still retains waves

$$
\begin{gathered}
\frac{\mathrm{d} w}{\mathrm{~d} t}=N_{u} b_{u} H_{u}+N_{s} b_{s} H_{s}, \\
\frac{\mathrm{d} b_{u}}{\mathrm{~d} t}+N_{u} w=0 \\
\frac{\mathrm{d} b_{s}}{\mathrm{~d} t}+N_{s} w=0
\end{gathered}
$$

The waves represented by (B10)-(B12) have different frequencies associated with the different phases $\left(b_{u}, b_{s}\right)$ and buoyancy parameters $\left(N_{u}, N_{s}\right)$. Phase boundaries are identified by the condition $b_{u}=b_{s}$, and the cloud indicator may be written as $H_{s}=$ $H\left(b_{s}-b_{u}\right)$.

For the asymptotic theory discussed in $\$ 5.2$, we consider the limiting dynamics for buoyancy frequencies $N_{u}, N_{s} \rightarrow \infty$, which is analogous to the limiting dynamics for $\epsilon^{-1} \rightarrow \infty$ in the fast-wave-averaging analysis of the moist Boussinesq system (2.43)-(2.48). We continue to use the special parameter setting $N_{u}{ }^{2}=2 N_{s}{ }^{2}$ as in the numerical simulations, where $N_{u}, N_{s}$ are both positive.

\section{REFERENCES}

Babin, A., Mahalov, A. \& Nicolaenko, B. 1996 Global splitting, integrability and regularity of 3d euler and Navier-Stokes equations for uniformly rotating fluids. Eur. J. Mech. B/Fluids 15 (3), 291-300. 


\section{Y. Zhang, L.M. Smith and S.N. Stechmann}

Babin, A., Mahalov, A. \& Nicolaenko, B. 1998 On nonlinear baroclinic waves and adjustment of pancake dynamics. Theor. Comput. Fluid Dyn. 11 (3-4), 215-235.

Babin, A., Mahalov, A. \& Nicolaenko, B. 2000 Fast singular oscillating limits and global regularity for the 3D primitive equations of geophysics. ESAIM: Math. Model. Numer. Anal. 34 (2), 201-222.

Babin, A., Mahalov, A., Nicolaenko, B. \& Zhou, Y. 1997 On the asymptotic regimes and the strongly stratified limit of rotating Boussinesq equations. Theoret. Comput. Fluid Dyn. 9 (3-4), 223-251.

BARTEllo, P. 1995 Geostrophic adjustment and inverse cascades in rotating stratified turbulence. J. Atmos. Sci. 52 (24), 4410-4428.

BennetTs, D.A. \& Hoskins, B.J. 1979 Conditional symmetric instability-a possible explanation for frontal rainbands. Q. J. R. Meteorol. Soc. 105 (446), 945-962.

Bousquet, A., Zelati, M.C. \& Temam, R. 2014 Phase transition models in atmospheric dynamics. Milan J. Maths 82 (1), 99-128.

BRETHERTON, C.S. 1987 A theory for nonprecipitating moist convection between two parallel plates. Part I: thermodynamics and "linear" solutions. J. Atmos. Sci. 44, 1809-1827.

Cao, Y., Hamouda, M., Temam, R., Tribbia, J. \& Wang, X. 2018 The equations of the multi-phase humid atmosphere expressed as a quasi variational inequality. Nonlinearity 31 (10), 4692-4723.

CAO, Z. \& CHO, H.-R. 1995 Generation of moist potential vorticity in extratropical cyclones. J. Atmos. Sci. 52 (18), 3263-3282.

Chen, S., Majda, A.J. \& Stechmann, S.N. 2015 Multiscale asymptotics for the skeleton of the Madden-Julian oscillation and tropical-extratropical interactions. Math. Clim. Weath. Forecast. 1, 43-69.

Chen, S., MAjda, A.J. \& Stechmann, S.N. 2016 Tropical-extratropical interactions with the MJO skeleton and climatological mean flow. J. Atmos. Sci. 73 (10), 4101-4116.

Cuijpers, J.W.M. \& DuYnKerke, P.G. 1993 Large eddy simulation of trade wind cumulus clouds. J. Atmos. Sci. 50 (23), 3894-3908.

Durran, D.R. \& Klemp, J.B. $1982 a$ The effects of moisture on trapped mountain lee waves. J. Atmos. Sci. 39 (11), 2490-2506.

Durran, D.R. \& Klemp, J.B. $1982 b$ On the effects of moisture on the Brunt-Väisälä frequency. J. Atmos. Sci. 39 (10), 2152-2158.

DutrifoY, A. \& MAJDA, A. 2006 The dynamics of equatorial long waves: a singular limit with fast variable coefficients. Commun. Math. Sci. 4 (2), 375-397.

Dutrifoy, A. \& MAJDA, A. 2007 Fast wave averaging for the equatorial shallow water equations. Commun. Part. Diff. Equ. 32, 1617-1642.

Dutrifoy, A., MAJdA, A.J. \& SCHochet, S. 2009 A simple justification of the singular limit for equatorial shallow-water dynamics. Commun. Pure Appl. Maths 62 (3), 322-333.

Edwards, T.K., Smith, L.M. \& Stechmann, S.N. 2020 a Atmospheric rivers and water fluxes in precipitating quasi-geostrophic turbulence. Q. J. R. Meteorol. Soc. 146, 1960-1975.

Edwards, T.K., Smith, L.M. \& Stechmann, S.N. 2020b Spectra of atmospheric water in precipitating quasi-geostrophic turbulence. Geophys. Astrophys. Fluid Dyn. 114, 715-741.

EMANUEL, K.A. 1979 Inertial instability and mesoscale convective systems. Part I: Linear theory of inertial instability in rotating viscous fluids. J. Atmos. Sci. 36 (12), 2425-2449.

Embid, P.F. \& MAJDA, A.J. 1996 Averaging over fast gravity waves for geophysical flows with arbitary potential vorticity. Comm. PDEs 21 (3-4), 619-658.

EMBID, P.F. \& MAJDA, A.J. 1998 Low Froude number limiting dynamics for stably stratified flow with small or finite Rossby numbers. Geophys. Astrophys. Fluid Dyn. 87 (1-2), 1-50.

Gill, A.E. 1982 Atmosphere-Ocean Dynamics. International Geophysics Series, vol. 30. Academic Press.

GrabOWSKI, W.W. \& MORRISON, H. 2008 Toward the mitigation of spurious cloud-edge supersaturation in cloud models. Mon. Weath. Rev. 136 (3), 1224-1234.

Grabowski, W.W. \& SmolarkiewicZ, P.K. 1996 Two-time-level semi-Lagrangian modeling of precipitating clouds. Mon. Weath. Rev. 124 (3), 487-497.

GreEnsPAN, H. 1969 On the nonlinear interaction of inertial modes. J. Fluid Mech. 36, 257-264.

Hernandez-Duenas, G., Majda, A.J., Smith, L.M. \& Stechmann, S.N. 2013 Minimal models for precipitating turbulent convection. J. Fluid Mech. 717, 576-611.

Hernandez-Duenas, G., Smith, L.M. \& Stechmann, S.N. 2014 Investigation of Boussinesq dynamics using intermediate models based on wave-vortical interactions. J. Fluid Mech. 747, 247-287.

HitTMEIR, S. \& KLEIN, R. 2018 Asymptotics for moist deep convection i: refined scalings and self-sustaining updrafts. Theor. Comput. Fluid Dyn. 32 (2), 137-164.

Hittmeir, S., Klein, R., Li, J. \& Titi, E.S. 2017 Global well-posedness for passively transported nonlinear moisture dynamics with phase changes. Nonlinearity 30 (10), 3676-3718. 


\section{Effects of clouds and phase changes on fast-wave averaging}

Hittmeir, S., KLEIN, R., Li, J. \& Titi, E.S. 2020 Global well-posedness for the primitive equations coupled to nonlinear moisture dynamics with phase changes. Nonlinearity 33, 3206-3236.

KESSLER, E. 1969 On the distribution and continuity of water substance in atmospheric circulations. Meteorological Monographs, vol. 32. American Meteorological Society.

Khouider, B., Majda, A.J. \& Stechmann, S.N. 2013 Climate science in the tropics: waves, vortices and PDEs. Nonlinearity 26 (1), R1-R68.

KLAinerman, S. \& MAJDA, A. 1981 Singular limits of quasilinear hyperbolic systems with large parameters and the incompressible limit of compressible fluids. Commun. Pure Appl. Maths 34 (4), $481-524$.

Klainerman, S. \& Majda, A. 1982 Compressible and incompressible fluids. Commun. Pure Appl. Maths 35 (5), 629-651.

KLEIN, R. \& MAJDA, A. 2006 Systematic multiscale models for deep convection on mesoscales. Theor. Comput. Fluid Dyn. 20, 525-551.

KUO, H.L. 1961 Convection in conditionally unstable atmosphere. Tellus 13 (4), 441-459.

Lelong, M.P. \& Riley, J.J. 1991 Internal wave-vortical mode interactions in strongly stratified flows. J. Fluid Mech. 232, 1-19.

LI, J. \& TiTi, E.S. 2016 A tropical atmosphere model with moisture: global well-posedness and relaxation limit. Nonlinearity 29 (9), 2674-2714.

LiN, Y.-L., FARley, R.D. \& ORVILLE, H.D. 1983 Bulk parameterization of the snow field in a cloud model. J. Clim. Appl. Meteor. 22 (6), 1065-1092.

LiU, X.-D., FEDKIw, R.P. \& KANG, M. 2000 A boundary condition capturing method for Poisson's equation on irregular domains. J. Comput. Phys. 160 (1), 151-178.

LIU, X.-D. \& SidERIS, T. 2003 Convergence of the ghost fluid method for elliptic equations with interfaces. Math. Comput. 72 (244), 1731-1746.

MajdA, A. 1984 Compressible Fluid Flow and Systems of Conservation Laws in Several Space Variables. Applied Mathematical Sciences, vol. 53. Springer.

MAJDA, A.J. 2003 Introduction to PDEs and Waves for the Atmosphere and Ocean. Courant Lecture Notes in Mathematics, vol. 9. American Mathematical Society.

MAJDA, A.J. 2007 Multiscale models with moisture and systematic strategies for superparameterization. J. Atmos. Sci. 64, 2726-2734.

MAjDA, A.J. \& EMBID, P. 1998 Averaging over fast gravity waves for geophysical flows with unbalanced initial data. Theor. Comput. Fluid Dyn. 11 (3-4), 155-169.

Majda, A.J. \& Souganidis, P.E. 2010 Existence and uniqueness of weak solutions for precipitation fronts: A novel hyperbolic free boundary problem in several space variables. Commun. Pure Appl. Maths 63 (10), 1351-1361.

Marquet, P. 2014 On the definition of a moist-air potential vorticity. Q. J. R. Meteorol. Soc. 140 (680), 917-929.

Marsico, D.H., Smith, L.M. \& Stechmann, S.N. 2019 Energy decompositions for moist Boussinesq and anelastic equations with phase changes. J. Atmos. Sci. 76, 3569-3587.

PAuluis, O. \& SCHUMACHER, J. 2010 Idealized moist Rayleigh-Bénard convection with piecewise linear equation of state. Commun. Math. Sci. 8, 295-319.

Phillips, O. 1968 The interaction trapping of internal gravity waves. J. Fluid Mech. 34, 407-416.

REMMEL, M. 2010 New models for the rotating shallow water and Boussinesq equations by subsets of mode interactions. PhD thesis, ProQuest LLC, Ann Arbor, MI, The University of Wisconsin - Madison.

Remmel, M. \& SMith, L.M. 2009 New intermediate models for rotating shallow water and an investigation of the preference for anticyclones. J. Fluid Mech. 635, 321-359.

ROGERS, R.R. \& YAU, M.K. 1989 A short course in cloud physics. Butterworth-Heinemann.

Rosemeier, J., Baumgartner, M. \& Spichtinger, P. 2018 Intercomparison of warm-rain bulk microphysics schemes using asymptotics. Maths Clim. Weath. Forecast. 4 (1), 104-124.

Schochet, S. 1994 Fast singular limits of hyperbolic PDEs. J. Differ. Equ. 114 (2), 476-512.

Schochet, S. 2005 The mathematical theory of low Mach number flows. ESAIM: Math. Model. Numer. Anal. 39 (3), 441-458.

Schubert, W.H., Hausman, S.A., Garcia, M., Ooyama, K.V. \& Kuo, H.-C. 2001 Potential vorticity in a moist atmosphere. J. Atmos. Sci. 58 (21), 3148-3157.

Schumacher, J. \& PAUluis, O. 2010 Buoyancy statistics in moist turbulent Rayleigh-Bénard convection. J. Fluid Mech. 648, 509-519.

SEIFERT, A. \& BEHENG, K.D. 2001 A double-moment parameterization for simulating autoconversion, accretion and selfcollection. Atmos. Res. 59, 265-281.

SEIfERT, A. \& BEHENG, K.D. 2006 A two-moment cloud microphysics parameterization for mixed-phase clouds. Part 1: Model description. Meteorol. Atmos. Phys. 92 (1-2), 45-66. 


\section{Y. Zhang, L.M. Smith and S.N. Stechmann}

Smith, L.M. \& Stechmann, S.N. 2017 Precipitating quasigeostrophic equations and potential vorticity inversion with phase changes. J. Atmos. Sci. 74, 3285-3303.

Smith, L.M. \& WALEFFE, F. 1999 Transfer of energy to two-dimensional large scales in forced, rotating three-dimensional turbulence. Phys. Fluids 11, 1608-1622.

SMith, L.M. \& WALEFFE, F. 2002 Generation of slow large scales in forced rotating stratified turbulence. J. Fluid Mech. 451, 145-168.

Sommeria, G. 1976 Three-dimensional simulation of turbulent processes in an undisturbed trade wind boundary layer. J. Atmos. Sci. 33 (2), 216-241.

Spyksma, K., Bartello, P. \& YAU, M.K. 2006 A Boussinesq moist turbulence model. J. Turbul. 7 (32), $1-24$.

Stechmann, S.N. \& Stevens, B. 2010 Multiscale models for cumulus cloud dynamics. J. Atmos. Sci. 67, 3269-3285.

Sukhatme, J., MajdA, A.J. \& Smith, L.M. 2012 Two-dimensional moist stratified turbulence and the emergence of vertically sheared horizontal flows. Phys. Fluids 24, 036602.

Tzou, C.-N. \& Stechmann, S.N. 2019 Simple second-order finite differences for elliptic PDEs with discontinuous coefficients and interfaces. Commun. Appl. Maths Comput. Sci. 14, 121-147.

VAllis, G.K. 2006 Atmospheric and Oceanic Fluid Dynamics: Fundamentals and Large-scale Circulation. Cambridge University Press.

Wetzel, A.N., Smith, L.M. \& Stechmann, S.N. 2017 Moisture transport due to baroclinic waves: linear analysis of precipitating quasi-geostrophic dynamics. Math. Clim. Weath. Forecast. 3 (1), $28-50$.

Wetzel, A.N., Smith, L.M. \& Stechmann, S.N. 2019a Discontinuous fronts as exact solutions to precipitating quasi-geostrophic equations. SIAM J. Appl. Maths 79, 1341-1366.

Wetzel, A.N., Smith, L.M., Stechmann, S.N. \& Martin, J.E. 2019b Balanced and unbalanced components of moist atmospheric flows with phase changes. Chin. Ann. Math. B 40, 1005-1038.

Wetzel, A.N., Smith, L.M., Stechmann, S.N., Martin, J.E. \& Zhang, Y. 2020 Potential vorticity and balanced and unbalanced moisture. J. Atmos. Sci. 77, 1913-1931.

Wingate, B.A., Embid, P., Holmes-Cerfon, M. \& TAYlor, M.A. 2011 Low Rossby limiting dynamics for stably stratified flow with finite Froude number. J. Fluid Mech. 676, 546-571.

Zelati, M.C., Frémond, M., Temam, R. \& Tribbia, J. 2013 The equations of the atmosphere with humidity and saturation: uniqueness and physical bounds. Physica D 264, 49-65.

Zelati, M.C., Huang, A., Kukavica, I., Temam, R. \& Ziane, M. 2015 The primitive equations of the atmosphere in presence of vapour saturation. Nonlinearity 28 (3), 625-668.

Zelati, M.C. \& TemAM, R. 2012 The atmospheric equation of water vapor with saturation. Bollettino dell'Unione Matematica Italiana 5 (2), 309-336.

Zhang, Y., Smith, L.M. \& Stechmann, S.N. 2021 Fast-wave averaging with phase changes: asymptotics and application to moist atmospheric dynamics. J. Nonlinear Sci. 31 (2), 38. 